Acta Crystallographica Section A

Foundations of Crystallography

ISSN 0108-7673

Received 31 July 2012

Accepted 16 January 2013

(C) 2013 International Union of Crystallography Printed in Singapore - all rights reserved

\section{Periodic entanglement II: weavings from hyperbolic line patterns}

\author{
Myfanwy E. Evans, ${ }^{a, b *}$ Vanessa Robins ${ }^{a}$ and Stephen T. Hyde ${ }^{a}$ \\ a'Department of Applied Mathematics, Research School of Physics, Australian National University, \\ Canberra, ACT 0200, Australia, and ${ }^{\mathbf{b}}$ Institut für Theoretische Physik, Friedrich-Alexander \\ Universität Erlangen-Nürnberg, Germany. Correspondence e-mail: \\ myfanwy.e.evans@physik.uni-erlangen.de
}

\begin{abstract}
Ordered arrays of cylinders, known as rod packings, are now widely used in descriptions of crystalline structures. These are generalized to include crystallographic packed arrays of filaments with circular cross sections, including curvilinear cylinders whose central axes are generic helices. A suite of the simplest such general rod packings is constructed by projecting line patterns in the hyperbolic plane $\left(\mathbb{H}^{2}\right)$ onto cubic genus-3 triply periodic minimal surfaces in Euclidean space $\left(\mathbb{E}^{3}\right)$ : the primitive, diamond and gyroid surfaces. The simplest designs correspond to 'classical' rod packings containing conventional cylindrical filaments. More complex packings contain three-dimensional arrays of mutually entangled filaments that can be infinitely extended or finite loops forming three-dimensional weavings. The concept of a canonical 'ideal' embedding of these weavings is introduced, generalized from that of knot embeddings and found algorithmically by tightening the weaving to minimize the filament length to volume ratio. The tightening algorithm builds on the SONO algorithm for finding ideal conformations of knots. Three distinct classes of weavings are described.
\end{abstract}

\section{Introduction}

Rod packings are mutually touching (i.e. packed) but nonintersecting crystalline arrays of straight cylinders in $\mathbb{E}^{3}$ (O'Keeffe \& Hyde, 1996). These include 'invariant rod packings, ${ }^{1}$ (Rosi et al., 2005). Since the earliest work on rod packings, a number of important patterns have been detected in the solid state (O'Keeffe \& Andersson, 1977; O'Keeffe, $1992)$ including the $A 15$ or $\beta$-tungsten $(\beta$-W) packing and a related packing of slightly lower density and symmetry, the $\beta$-Mn packing. More recently, O'Keeffe and colleagues have provided a systematic technique for enumerating conventional rod packings and they have come up with a number of new examples (O'Keeffe et al., 2001, 2002; Rosi et al., 2005).

In the course of our own investigations of spatial patterns, we have found a novel route to many of these rod packings, involving projections into $\mathbb{E}^{3}$ of non-intersecting geodesics from the two-dimensional hyperbolic plane $\left(\mathbb{H}^{2}\right)$. These packings of non-intersecting geodesics are closely related both to standard tilings (Ramsden et al., 2009) and free tilings (Evans et al., 2013) of $\mathbb{H}^{2}$. This approach also yields many more complex arrays of curvilinear rods that wind through space forming generic helices. These arrays share many of the

\footnotetext{
${ }^{1}$ An invariant rod packing is composed of non-overlapping rods (cylinders) which correspond to invariant line positions of the space groups, so that the rods lie along the directions of non-intersecting symmetry axes (O'Keeffe et al., 2001; Rosi et al., 2005).
}

features of conventional rod packings. Our generalized rod packings are constructed from two-dimensional tilings of hyperbolic space $\left(\mathbb{H}^{2}\right)$ and signal a further break from rectilinear geometries. The approach shares many of the techniques described in detail in a companion paper (Evans et al., 2013) and elsewhere (Ramsden et al., 2009; Evans \& Hyde, 2011; Castle et al., 2011, 2012).

The admission of curvilinear rods leads to the following possibilities. First, the rods can close up on themselves, forming finite loops rather than infinitely extended components. Our approach allows the catenation of those loops to be varied, forming, for example, 'chainmail' structures. Second, the packings can contain complex entanglements of the filaments. It is intuitively clear that entanglement can be an essential contributor to the material properties of the packing, since mutual winding of adjacent filaments can dramatically alter both the bulk modulus and Poisson's ratio of a structure.

\section{Weavings in $\mathbb{E}^{3}$}

We construct three-dimensional crystalline arrays of curvilinear space curves (or filaments), called weavings. The weavings discussed here emerge from two-dimensional geodesic arrays on the simplest cubic TPMSs (triply periodic minimal surfaces), namely the now well known $P$ (primitive), $D$ (diamond) and $G$ (or gyroid) (Fogden \& Hyde, 1992). Systematic enumeration of suitable two-dimensional arrays 
on the TPMSs, using an extension of Delaney-Dress tiling theory to so-called 'free tilings', allows us to catalogue and describe weavings as described below. Generalized rod packings are the set of mutually tangential canal surfaces of the filaments, formed by the envelope of spheres centred on the filament with both curvature and torsion (Hilbert \& Cohn-Vossen, 1952), and we retain the original meaning of the term 'rod packing' to describe arrays of straight cylinders only.

The construction proceeds by decorating tiles of $\mathbb{H}^{2}$ with line segments of the hyperbolic pattern, then mapping the decorated tile to the TPMS, giving a (generally curved) edge segment wrapped on the TPMS. The technique employs aspects of group theory, topology and hyperbolic geometry. A detailed account of the technique and the enumeration process for conventional tilings has been given elsewhere (Ramsden et al., 2009; Robins et al., 2004). Those patterns are built from tessellations of $\mathbb{H}^{2}$ by (simply connected) tiles of finite area. These tiles map to closed cycles on the TPMS and conventional one-component nets in $\mathbb{E}^{3}$ (Ramsden et al., 2009).

Free tilings result from tessellations by (simply connected) tiles whose areas and edge lengths are unbounded. In the companion paper to this one (Evans et al., 2013), we introduced free 'ribbon tilings' that are tessellations of $\mathbb{E}^{2}$ or $\mathbb{H}^{2}$ by infinite strip-like tiles. The forms of infinite tiles can be most easily classified from their associated 'medial axes', defined as the centres of discs (whose diameter is equal to the tile width) whose union describes the tiles. The medial axes of ribbon tiles are unbranched geodesics; medial axes of branched-ribbon tiles are nets, or branched geodesics.

Branched-ribbon tilings emerged from an earlier study of tree-like graphs in the hyperbolic plane (Hyde \& Oguey, 2000). In that work, we noticed that the convex hulls of the trees form arrays of (hyper-)parallel geodesics and these can be considered as edges of symmetric tessellations of $\mathbb{H}^{2}$. In contrast to ribbon tilings, these tessellations of $\mathbb{H}^{2}$ have no counterpart in $\mathbb{E}^{2}$ : each tile is bounded by an infinite number of hyperparallel edges, yet is vertex-free. The medial axes of these branched-ribbon tiles coincide with the hyperbolic trees [that form edges of ribbon tilings discussed in the companion paper (Evans et al., 2013)]. Branched-ribbon tilings are therefore closely related to ribbon tilings. (Note, however, that the width of branched-ribbon tiles varies. Their medial axes are defined by centres of 'maximal' discs, that are wedged between tile edges. The discs are large enough that any larger disc necessarily includes some points outside the tile.)

Here we analyse the simplest members of these branchedribbon tilings, namely the regular examples, that, like Platonic polyhedra, have symmetrically identical edges and faces. In the language of tiling theory, these (vertex-free) patterns are edge- and face- 1 transitive in $\mathbb{H}^{2}$ (and on the TPMS). The tile edges (which are geodesics in $\mathbb{H}^{2}$ ) map to one-dimensional spatial curves in $\mathbb{E}^{3}$. An infinite variety of regular branchedribbon tilings is possible, whose edges map to $\mathbb{E}^{3}$ to form a variety of crystalline arrays of identical curvilinear forms, defining the filaments of the weaving.

\subsection{A taxonomy of weavings}

These branched-ribbon tilings project onto the TPMS to form two distinct topologies. Generic examples contain infinite curvilinear lines, resulting in weavings made up of infinite filaments. In some cases, the filaments form finite closed loops and the weaving degenerates to a periodic array of catenated loops, built of links (Cromwell, 2004). We include a brief discussion of one such example, separately.

We have found three distinct classes of weavings, distinguished by their behaviour on straightening the curvilinear filaments in three-dimensional space, $\mathbb{E}^{3}$ (Evans \& Hyde, 2011). Define a filament axis as the straight line that minimizes the sum of the (Euclidean) distances from points on the filament to the rectilinear axis. For example, the axis of an ideal helical filament is coincident with the central screw axis of the helix. A continuous motion from the original helix to its filament axis is therefore akin to straightening a helix along its central axis. In general, our filaments are not ideal helices, since their specific form depends on the spatial trajectory of the filament on the TPMS. However, the filaments are by construction translationally periodic (cf. Evans et al., 2013); therefore, their axes are necessarily parallel to their vector of translational periodicity. Our weaving taxonomy depends on the behaviour of the weaving as the filaments morph from their initial curvilinear filament geometry to the final state, where all filaments coincide with their rectified filament axes. We classify weavings according to the changes (if any) of filament entanglements during this process.

The notion of entanglement is borrowed, somewhat loosely, from knot theory. Distinct knots cannot be interconverted without edges passing through each other. Conversely, equivalent knots can be interconverted without these 'phantom moves' and mappings of their complementary volumes are ambient isotopic (Cromwell, 2004). Similarly, equivalent entanglements of a net are related by an ambient isotopy of their complementary volumes; these are called equivalent isotopes. By analogy, generalized rod packings are labelled as equivalent isotopes if they can be interconverted without phantom moves. Given a generalized rod packing, we define its 'untangled' isotope to be that formed by rods centred on the filament axes of the initial weaving. (We clarify that for special cases where the rods intersect below.) Here we avoid subtle questions of ambient isotopy associated with rod packings and analyse the possible interconversions of rod packings via specific transformations only (rectification from curvilinear to straight rods and tightening, described below). If phantom moves are present during rectification, the initial and final packings are distinct isotopes, with distinct entanglements. In that case, the initial weaving is tangled.

The simplest class of weaving ('class I') is untangled. The filaments can be rectified from their (generically) curvilinear filament geometry - inherited from the edges on the TPMS to form straight filaments that coincide with their filament axes, without filaments sharing common points in space at any stage during this rectification process. The generalized rod packing formed from the weaving is therefore an equivalent 
isotope to the rectilinear (untangled) rod packing, so it too is necessarily untangled. Untangled weavings include conventional rod packings, both cubic and anisotropic invariant rod packings (Rosi et al., 2005; O'Keeffe et al., 2001). In addition, this class includes other invariant rod packings of various symmetries and generalized rod packings, with curvilinear rods.

A second class ('II') is relevant to weavings where the rectification of all filaments to their straight filament axes results in intersections. In those cases, the untangled state is not uniquely defined, as the straight filaments form a net whose vertices are common to more than one filament. The intersection can be removed by two possible perturbations at each 'vertex': lifting one filament to pass over the other, or under. Here we define the untangled weaving to be the infinite family of cases formed by any combination of those moves. (These intersections are neither tangled nor untangled, yet share features of both states.) If the initial curvilinear weaving can be 'rectified' into any one of those untangled embeddings without phantom moves, it is itself untangled and is a class II weaving. This implies that the weaving can be transformed from its initial state to (intersecting) straight filaments aligned with the filament axes without edges passing through each other and reversing edge crossings; however, edges just touch in their final rectified configuration.

The third class ('III') collects weavings whose filaments are sufficiently tangled to impede each other from rectification along their filament axes without filaments passing through each other, changing the entanglement of the weaving. Here we assume the fully rectified rod packing is not intersecting. We use the untangled isotope to help describe the original weaving, but emphasize that they are not equivalent under ambient isotopy. Weavings in this class are tangled versions of their rectified rod packings.

We assign names to weavings as follows. Untangled weavings in class I are given the name of their associated rectified rod packing. These are often among the set of 14 invariant rod packings enumerated in Rosi et al. (2005), in which case we use the names derived from a related lattice complex of the space group from which they are constructed. These are $\Pi^{+}, \Pi^{*}, \Sigma^{+}$, $\Gamma, \Omega^{+}, \Sigma^{*}$, where the + or - superscript denotes one enantiomer of the packing and the $*$ exponent the intergrowth of both + and - enantiomers (O'Keeffe et al., 2001). The other eight invariant rod packings from this enumeration are identified by their structure number (\#1-\#8), as given in Rosi et al. (2005). Where the weavings are related to invariant rod packings of other symmetry, they are given a label 'Rod (tetr.)' which reflects that they are related to a rod packing with tetragonal space-group symmetry, or 'Rod (tri.)' where they have trigonal space-group symmetry.

Class II weavings, whose filaments (just) intersect on straightening, are labelled 'Intersect (net)' where net denotes the three-letter code for the net formed by the intersecting filaments, as listed in the RCSR database (O'Keeffe et al., 2008).

Class III 'tangled' weavings are named according to the associated untangled rod packing corresponding to the arrangement of filament axes (as per type I weavings). This rod-packing label is prepended by the term 'tangled' to indicate that it is not ambient isotopic to the rod packing specified.

Lastly, we name the examples whose filaments form finite closed loops as 'loops'.

\subsection{Ideal embeddings}

General weavings composed of curvilinear filaments share many features with knots. Standard rod packings have a unique geometry or embedding in $\mathbb{E}^{3}$, whereas generalized rod packings do not, since the curvilinear filament geometry can usually vary without changing the entanglement of the associated generalized rod packing. Like knots, isotopes are flexible. It is therefore helpful to describe a canonical embedding of each isotope. The route that we adopt here builds on the concept of a 'tight embedding' from knot theory that often though not always - affords a unique and therefore canonical embedding for conventional knots and links (Stasiak et al., 1998).

Tight or 'ideal' embeddings of knots minimize the knot length for a given diameter $(L / D)$. A fast and effective numerical algorithm is the SONO algorithm (Pieranski, 1998). Here we adopt an extended version of this concept to find ideal tight conformations of periodic weavings, using a generalized version of the SONO algorithm to allow for periodic boundary conditions and minimize $L / D$ within one unit cell. This adapted algorithm, introduced in the companion paper (Evans et al., 2013), is explored in detail in Evans (2011) and we refer to it as PB-SONO throughout this paper.

The ideal embedding of a (periodic) generalized rod packing is defined to be the embedding that minimizes $L / D$ within a unit cell of the structure: $L / D$ is a dimensionless measure of the filament length per unit cell normalized by the filament diameter. This measure depends on the unit cell and does not always offer a useful index of comparative tightness. We therefore also characterize generalized rod packings by their packing fraction, which is independent of the unit-cell shape and volume $(V):\left(\pi L D^{2}\right) / 4 V$. We note, however, that this value may not be maximized in an ideal embedding, as we will see in examples explored later in this paper. In other words, ideal embeddings are not necessarily the densest.

\section{Regular branched-ribbon tilings}

The concept of free tilings has been introduced in the companion paper (Evans et al., 2013). Here too we explore regular free tilings that are vertex- 1 , edge- 1 and tile- 1 transitive. Details of the representation of free tilings by DelaneyDress symbols are given in Evans et al. (2013). Here we focus on regular tilings by branched ribbons whose tile edges form arrays of infinite, vertex-free lines in $\mathbb{H}^{2}$. Recall that those arrays are in one-to-one correspondence with the free ribbon tilings: they are the medial axes of the related ribbon free tiling, and vice versa. That correspondence simplifies enumeration of regular branched-ribbon tilings. 
Consider first the free tilings of $\mathbb{H}^{2}$. The fundamental regions for free tilings with threefold, fourfold and sixfold symmetry are shown in Fig. 1. Tilings are named according to their group number in the $\star 246 / 0$ o o quotient group (Robins et al., 2004; Evans et al., 2013), appended with ' $R L$ ', signifying the regular line arrays characteristic of branched-ribbon tilings (cf. Evans et al., 2013).

To project to the TPMS, we first embed these tiles into the $\star 246$ tiling of $\mathbb{H}^{2}$. A systematic enumeration of these embeddings is given in Evans et al. (2013) and we refer the reader to this paper for further details. In short, the embedding can be specified by the hyperbolic length of one asymmetric unit of the line (tile boundary) when embedded in $\mathbb{H}^{2}$. We use that length to label the tiling patterns. A complete table of these embedded tilings is given in Appendix $A$.

A rich variety of filament arrays is formed in $\mathbb{E}^{3}$ by projecting the edges of regular branched-ribbon tilings onto the $P, D$ and $G$ (yroid) TPMS. These result in generalized rod packings of types I, II and III as well as catenated links. A complete enumeration of the weavings is given in Appendix $B$, and crystallographic data for all weavings are given as supplementary material. ${ }^{2}$

\section{Class I weavings}

\subsection{Invariant rod packings: parallel and layers}

There are eight invariant rod packings composed of parallel rods or layers of rods enumerated in Rosi et al. (2005); four of these are composed solely of parallel rods and the other four composed of stacked layers, with parallel rods in each layer. Four of these rod packings, \#1, \#2, \#3 and \#6, arise via branched-ribbon tilings on the $P, D$ and $G$ surfaces.

The \#1 rod packing (Rosi et al., 2005) consists of a twodimensional hexagonal arrangement of parallel rods whose axes lie at the vertices of the $\{6,3\}$ planar tiling and is a close-packed array of discs. A number of regular branchedribbon tilings from the $P, D$ and $G$ surfaces lead to filament arrays whose average axes form the same pattern as the filaments of the $\# 1$ rod packing. These are the $P_{122 R L}\left(\cosh ^{-1}(3 / 2)\right), P_{93 R L}\left(\cosh ^{-1}(2 \sqrt{2})\right), D_{122 R L}\left(\cosh ^{-1}(5 / 2)\right)$

\footnotetext{
${ }^{2}$ Supplementary material for this paper is available from the IUCr electronic archives (Reference: EO5020). Services for accessing these data are described at the back of the journal.
}

Figure 1

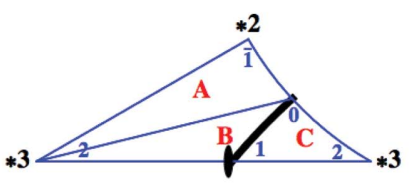

129RL

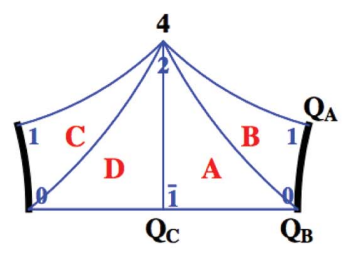

114RL

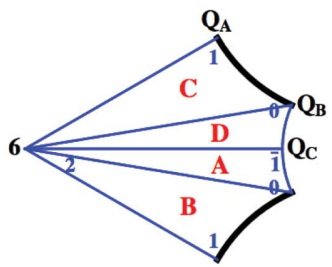

$93 \mathrm{RL}$

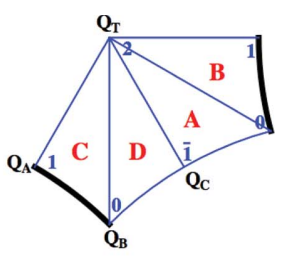

118RL
Delaney-Dress symbols for the regular three-, four- and sixfold branched-ribbon tilings with infinite geodesic boundaries. The tilings are named according to their group number in the $\star 246 / \circ \circ \circ$ quotient group (Robins et al., 2004; Evans et al., 2013).

and $G_{122 R L}^{+}\left(\cosh ^{-1}(3 / 2)\right)$ patterns. For example, the $G_{122 R L}^{+}\left(\cosh ^{-1}(3 / 2)\right)$ pattern is achiral, with helical filaments, alternating between left- and right-handed enantiomers, as shown in Fig. 2. Another variant of this rod packing is the structure $G_{122 R L}^{+}\left(\cosh ^{-1}(5 / 2)\right)$, which has double helices along each rod axis.

A two-dimensional square array of parallel rods whose axes are located at the vertices of a $\{4,4\}$ tiling of $\mathbb{E}^{2}$ constitutes the \#2 rod packing (Rosi et al., 2005). Structures that are equivalent to this packing are $\quad P_{123 R L}\left(\cosh ^{-1}(\sqrt{2})\right), \quad D_{123 R L}\left(\cosh ^{-1}(\sqrt{3} / \sqrt{2})\right)$, $D_{114 R L}\left(\cosh ^{-1}(3 \sqrt{3} / \sqrt{2})\right), \quad G_{123 R L}^{+}\left(\cosh ^{-1}(\sqrt{3} / \sqrt{2})\right)$, $G_{114 R L}^{+}\left(\cosh ^{-1}(\sqrt{3})\right)$ and $G_{114 R L}^{-}\left(\cosh ^{-1}(3 \sqrt{3})\right)$. Fig. 2 shows the tetragonal $P_{123 R L}\left(\cosh ^{-1}(\sqrt{2})\right)$ pattern (space group $I 4 / \mathrm{mmm}$ ), which consists of undulating rods. Another structure, $P_{114 R L}\left(\cosh ^{-1}(2 \sqrt{2})\right)$, is related to the \#2 rod packing and is composed of quadruple helices along the rod axes. Similarly, the $G_{114 R L}^{+}\left(\cosh ^{-1}(2 \sqrt{2})\right)$ structure has triple helices along the rod axes. Further, tangled versions of the \#2 rod packing arise as structures $D_{114 R L}\left(\cosh ^{-1}(2 \sqrt{2})\right)$ and $G_{114 R L}^{-}\left(\cosh ^{-1}(2 \sqrt{2})\right)$, see $\$ 6$.

The \#3 rod packing consists of parallel rods whose axes are at vertices of a trigonal (3.6.3.6) tiling. The $G_{93 R L}\left(\cosh ^{-1}(\sqrt{2})\right)$ pattern, shown in Fig. 2, has identical average axes to this rod packing. The trigonal curvilinear array has undulating filaments (and space group $R \overline{3} c$ ).

Stacked layers of parallel rods where the filament axes of adjacent layers are orthogonal comprise the \#6 rod packing. 


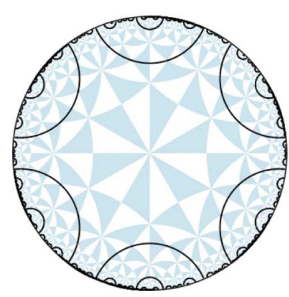

*246 $6_{122 R L}\left(\cosh ^{-1}\left(\frac{3}{2}\right)\right)$

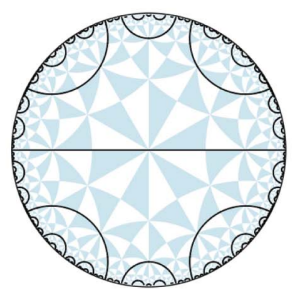

$* 246_{123 R L}\left(\cosh ^{-1}(\sqrt{2})\right)$

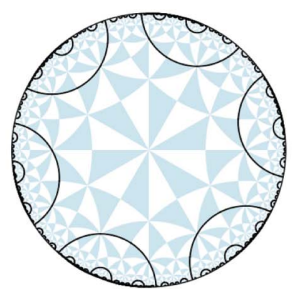

$* 246{ }_{93 R L}\left(\cosh ^{-1}(\sqrt{2})\right)$

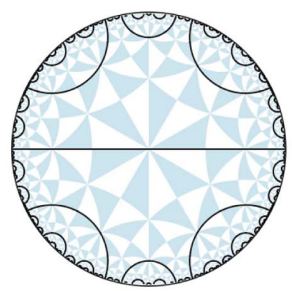

$* 246_{123 R L}\left(\cosh ^{-1}(\sqrt{2})\right)$

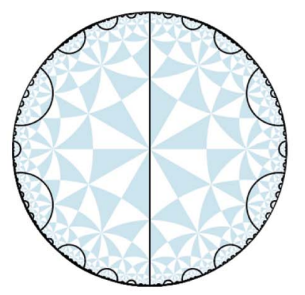

$2 * 222$ symmetry

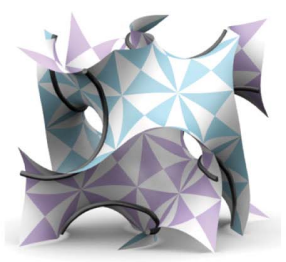

$G_{122 R L}^{+}\left(\cosh ^{-1}\left(\frac{3}{2}\right)\right)$

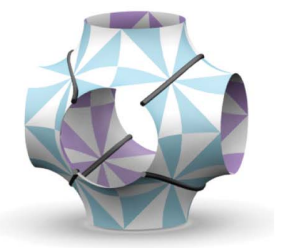

$P_{123 R L}\left(\cosh ^{-1}(\sqrt{2})\right)$

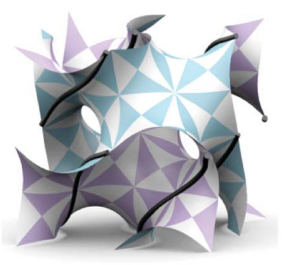

$\left.G_{93 R L}^{+}\left(\cosh ^{-1} \sqrt{2}\right)\right)$

undulating \#3 rod packing

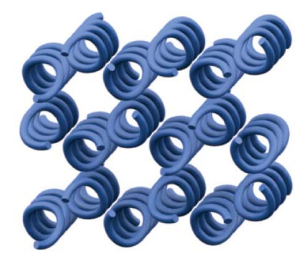

helical \#1 rod packing

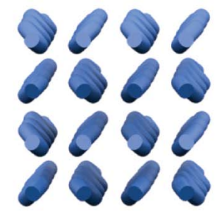

undulating \#2 rod packing
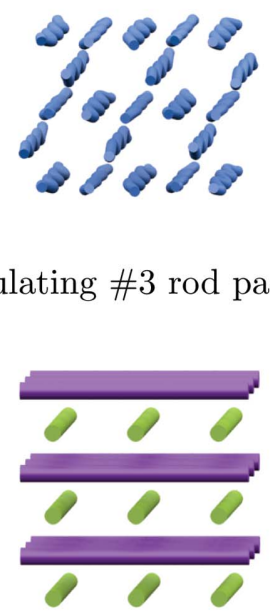

\#6 rod packing

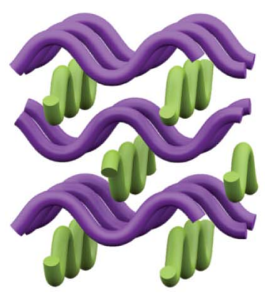

\#5 rod packing

Figure 2

Invariant rod packings: parallel and layers. These are shown as tilings of $\mathbb{H}^{2}$, tilings of the TPMS on which they are built and as filament packings in three-dimensional space. The names of the structures are given below each image.

Structures related to this packing are $D_{123 R L}\left(\cosh ^{-1}(\sqrt{2})\right)$ and $G_{114 R L}^{-}\left(\cosh ^{-1}(\sqrt{3})\right)$. Fig. 2 shows the former, which has space group $\mathrm{P}_{2} / \mathrm{mmc}$.

[The \#4, \#5, \#7 and \#8 rod packings described by O'Keeffe et al. have not emerged from the current enumeration of regular $\mathbb{H}^{2}$ patterns. Further examples are sure to emerge on more complete enumeration of hyperbolic patterns. For example, a structure related to the \#5 rod packing, composed of stacked layers at half the density of the \#6 rod packing, forms on the $D$ surface by a branched-ribbon tiling with $2 \star 222$ symmetry (group 104). The tiling is edge-2 transitive and therefore irregular. The structure has undulating components and space group $I 4_{1} /$ amd.]

All of the packings with parallel and layered rods converge to a common rod packing on tightening using the PB-SONO algorithm, namely the \#4 rod packing (Fig. 3). They are therefore all equivalent isotopes. Those patterns with parallel average axes (\#1-\#4) tighten by straightening and rearrangement of the rods to form the dense two-dimensional hexagonal conformation. Similarly, the patterns composed of stacked layers of parallel rods (\#5\#8) also eventually tighten to form this ideal conformation by rotation of every alternate layer to form parallel axes, as expected. Although various initial configurations derived from the TPMS branched-ribbon tilings have distinct initial unit cells, they further symmetrize on tightening, forming a common primitive unit cell containing just one rod. The packing fraction is $\pi /(2 \sqrt{3}) \simeq 0.91$ regardless of the choice of unit cell. The $L / D$ measure depends on the unit cell and for the smallest unit cell, which contains only one $\operatorname{rod}, L / D=1$.

\subsection{Invariant rod packings: cubic examples}

Six invariant rod packings of cubic symmetry are enumerated in Rosi et al. (2005). Generalized rod packings related to five of these six arise from regular branched-ribbon tilings of $\mathbb{H}^{2}$; the sixth does not have a hyperbolic antecedent corresponding to the regular tiling of branched ribbons.

The filament axes of the $G_{124 R L}^{+}\left(\cosh ^{-1}(\sqrt{3} / \sqrt{2})\right)$ pattern align with the $\Pi^{+}$rod packing (Fig. 4). The pattern that emerges from the tiling on the $G$ consists of close to ideal helical filaments, all of equivalent chirality. [The $G_{124 R L}^{-}\left(\cosh ^{-1}(\sqrt{3} / \sqrt{2})\right)$ tiling, which is the $G$ surface fibration of the same tiling by the second covering map of the $G$ surface (Evans et al., 2013), gives the $\Pi^{-}$enantiomer of the chiral rod packing.] The ideal embedding of this pattern has curvilinear filaments with close 

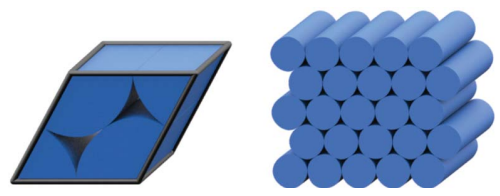

Figure 3

The ideal configuration for all weavings composed of parallel rods, or stacked layers of parallel rods. One unit cell is shown, which contains a single rod and has lattice parameters $(1,1,1, \pi / 2, \pi / 2, \pi / 3)$. The length of the rod is 1 , the diameter is also 1 and the volume of the unit cell is $\sqrt{3} / 2$. Thus $L / D=1$ and the packing fraction is approximately 0.91 .
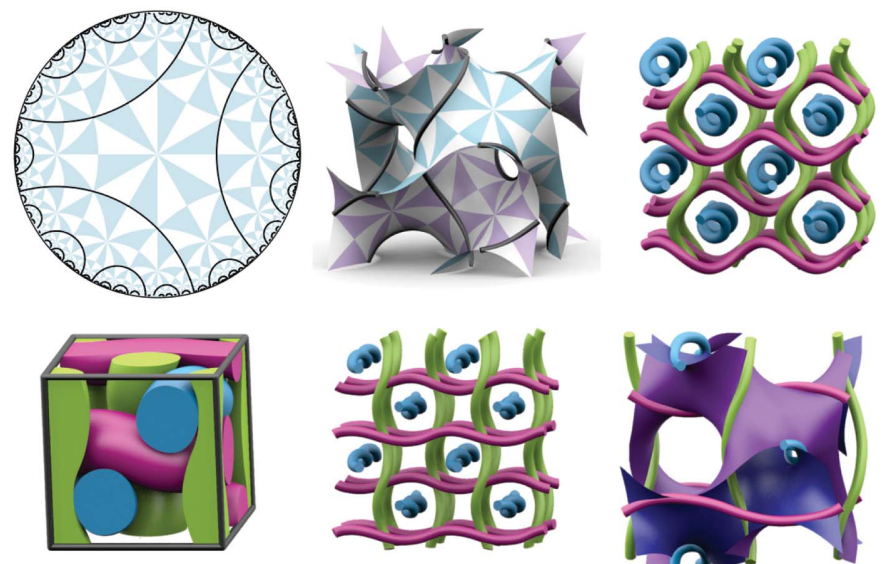

Figure 4

The $G_{124 R L}^{+}\left(\cosh ^{-1}(\sqrt{3} / \sqrt{2})\right)$ structure: a helical $\Pi^{+}$rod packing. (Top) Geometry of the decoration, given in $\mathbb{H}^{2}$, on the $G$ surface and in $\mathbb{E}^{3}$. (Bottom) The ideal configuration in one unit cell, in $\mathbb{E}^{3}$ and on a surface parallel to the $G$ surface such that one channel has been deflated and the other enlarged.

to helical trajectories and is formed by tightening both straight or curved starting filaments. Remarkably, the helices of this ideal structure decorate a surface parallel to the $G$ minimal surface such that one channel is slightly deflated and the other is enlarged, as shown in Fig. 4, indicating the underlying relevance of the $G$ TPMS to this pattern. The $L / D$ value for this conformation is 17.91 and the packing fraction is 0.66 .

The $D_{124 R L}\left(\cosh ^{-1}(\sqrt{3} / \sqrt{2})\right)$ tiling has straight rods, coincident with the $\Pi^{*}$ rod packing, an intergrowth of both chiral enantiomers of $\Pi^{+}$and $\Pi^{-}$. Its ideal configuration also consists of straight rods, with $L / D=6$ and packing fraction $(3 \pi) / 16 \simeq 0.59$ (Fig. 5).

A $G_{129 R L}^{+}\left(\cosh ^{-1}(3 / 2)\right)$ tiling results in quasi-helical filaments winding on the $G$ surface, where all the helices have
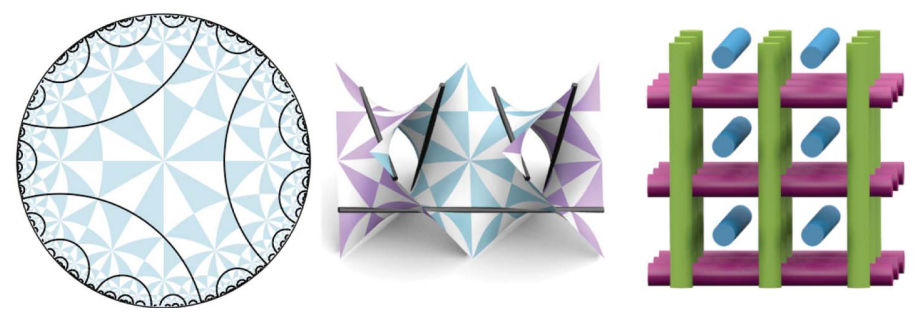

Figure 5

$D_{124 R L}\left(\cosh ^{-1}(\sqrt{3} / \sqrt{2})\right)$, equivalent to the $\Pi^{*}$ rod packing. It is shown (from left to right) in $\mathbb{H}^{2}$, on the $D$ surface, in $\mathbb{E}^{3}$ and as an ideal configuration within one unit cell.
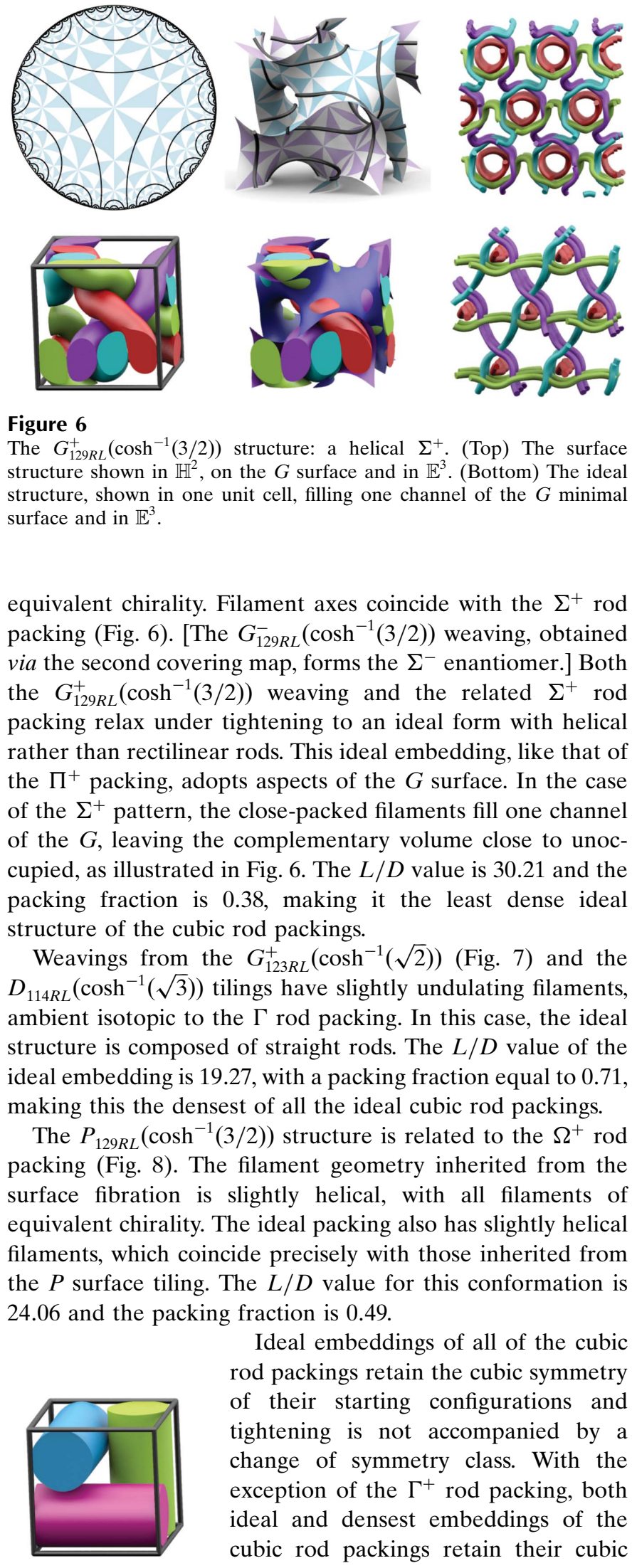

Figure 6

The $G_{129 R L}^{+}\left(\cosh ^{-1}(3 / 2)\right)$ structure: a helical $\Sigma^{+}$. (Top) The surface structure shown in $\mathbb{H}^{2}$, on the $G$ surface and in $\mathbb{E}^{3}$. (Bottom) The ideal structure, shown in one unit cell, filling one channel of the $G$ minimal surface and in $\mathbb{E}^{3}$.

equivalent chirality. Filament axes coincide with the $\Sigma^{+}$rod packing (Fig. 6). [The $G_{129 R L}^{-}\left(\cosh ^{-1}(3 / 2)\right)$ weaving, obtained via the second covering map, forms the $\Sigma^{-}$enantiomer.] Both the $G_{129 R L}^{+}\left(\cosh ^{-1}(3 / 2)\right)$ weaving and the related $\Sigma^{+} \operatorname{rod}$ packing relax under tightening to an ideal form with helical rather than rectilinear rods. This ideal embedding, like that of the $\Pi^{+}$packing, adopts aspects of the $G$ surface. In the case of the $\Sigma^{+}$pattern, the close-packed filaments fill one channel of the $G$, leaving the complementary volume close to unoccupied, as illustrated in Fig. 6 . The $L / D$ value is 30.21 and the packing fraction is 0.38 , making it the least dense ideal structure of the cubic rod packings.

Weavings from the $G_{123 R L}^{+}\left(\cosh ^{-1}(\sqrt{2})\right.$ ) (Fig. 7) and the $D_{114 R L}\left(\cosh ^{-1}(\sqrt{3})\right)$ tilings have slightly undulating filaments, ambient isotopic to the $\Gamma$ rod packing. In this case, the ideal structure is composed of straight rods. The $L / D$ value of the ideal embedding is 19.27 , with a packing fraction equal to 0.71 , making this the densest of all the ideal cubic rod packings.

The $P_{129 R L}\left(\cosh ^{-1}(3 / 2)\right)$ structure is related to the $\Omega^{+}$rod packing (Fig. 8). The filament geometry inherited from the surface fibration is slightly helical, with all filaments of equivalent chirality. The ideal packing also has slightly helical filaments, which coincide precisely with those inherited from the $P$ surface tiling. The $L / D$ value for this conformation is 24.06 and the packing fraction is 0.49 .

Ideal embeddings of all of the cubic rod packings retain the cubic symmetry of their starting configurations and tightening is not accompanied by a change of symmetry class. With the exception of the $\Gamma^{+}$rod packing, both ideal and densest embeddings of the cubic rod packings retain their cubic symmetry, where any deformation of the unit cell from its cubic form both increases $L / D$ and decreases the packing fraction. 

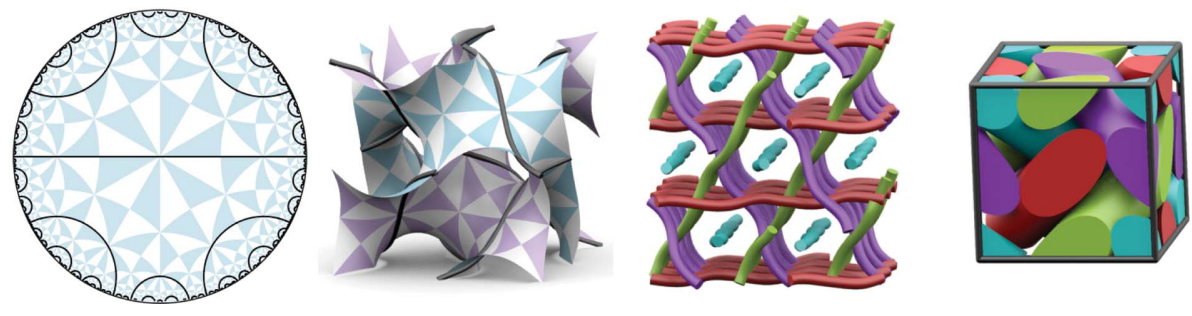

Figure 7

The $G_{123 R L}^{+}\left(\cosh ^{-1}(\sqrt{2})\right)$ structure, equivalent to the $\Gamma$ rod packing. The structure is shown (from left to right) in $\mathbb{H}^{2}$, on the $G$ surface, in $\mathbb{E}^{3}$ and as an ideal structure in one unit cell.
$G$ minimal surface and the second ideal enantiomer fills the other channel. The correspondence between the TPMS and ideal embeddings of these cubic rod packings is curious, given that tight embeddings are not a priori related to two-dimensional hyperbolic patterns. Among all cases, only the ideal embedding of the $\Gamma$ rod packing does not relate readily to the geometry of the $P, D$ or $G$ morphologies.

When different lattice parameters are imposed for the $\Gamma^{+}$ $\left[G_{123 R L}^{+}\left(\cosh ^{-1}(\sqrt{2})\right)\right]$ pattern, and each of these structures then tightened given this input, we see an interesting result. Increasing the $c$ axis (elongating the cube cell to be of size $1 \times 1 \times c)$ results in ideal embeddings with a higher $L / D$ value and a higher packing fraction, forming denser packings, that are, however, looser than the cubic case. These denser, looser embeddings have undulating filaments, with ever larger curvature variations as $c$ is increased.

These regular tilings produce generalized rod packings whose ideal forms coincide with five of the six invariant cubic rod packings detailed in O'Keeffe et al. (2001). The sixth of these rod packings is the $\Sigma^{*}$ packing, which is an interwoven variant containing a $\Sigma^{+}$and a $\Sigma^{-}$(an enantiomeric pair). It does not emerge from regular branched-ribbon tilings on the cubic TPMS. (It is probable, however, that this structure is an irregular case, related to a lower-symmetry orbifold than those of regular free tilings.) It is interesting to note that the ideal embedding of the $\Sigma^{*}$ rod packing is exactly equivalent to the interweaving of the ideal $G_{129 R L}^{+}\left(\cosh ^{-1}(3 / 2)\right)$ and $G_{129 R L}^{-}\left(\cosh ^{-1}(3 / 2)\right)$ structures (related to the $\Sigma^{+}$and $\Sigma^{-}$ packings). Remarkably, the complementary volume to the ideal $G_{129 R L}^{+}\left(\cosh ^{-1}(3 / 2)\right)$ structure [or equivalently the $G_{129 R L}^{-}\left(\cosh ^{-1}(3 / 2)\right)$ structure] is just suitable to accommodate the opposite enantiomer, also in its ideal form. In other words, the ideal $G_{129 R L}^{+}\left(\cosh ^{-1}(3 / 2)\right)$ structure fills one channel of the
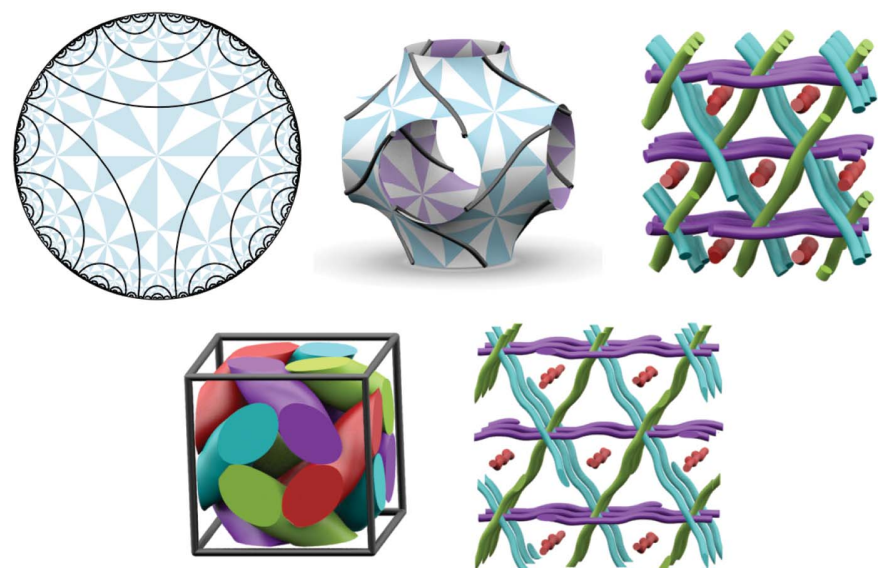

Figure 8

The $P_{129 R L}\left(\cosh ^{-1}(3 / 2)\right)$ structure, related to the $\Omega^{+}$rod packing. (Top) The surface fibration, shown in $\mathbb{H}^{2}$, on the surface and in $\mathbb{E}^{3}$. (Bottom) The ideal configuration, shown in one unit cell and in $\mathbb{E}^{3}$. The ideal structure is very close to the surface fibration.

\subsection{Invariant rod packings: non-cubic examples}

The enumeration of weavings from regular branchedribbon tilings also results in five additional tetragonal and trigonal patterns, whose filament axes coincide with rectilinear, invariant rod packings not enumerated in O'Keeffe et al. (2001) and Rosi et al. (2005).

The $P_{114 R L}\left(\cosh ^{-1}(\sqrt{3})\right)$ structure is shown in Fig. 9. The filament geometry inherited from the surface fibration consists of a tetragonal array of straight lines, where the four filaments within a unit cell are described by the trajectories $\left\{u, 0, \frac{1}{2}+u\right\}$, $\left\{u, \frac{1}{2},-u\right\},\left\{\frac{1}{2}, u, \frac{1}{2}+u\right\}$ and $\{0, u,-u\}$ within the space group $P 4 / n n c$. In the ideal conformation of this rod packing the filaments deviate slightly from their rod axes, forming undulating trajectories. The minimum $L / D$ value, 15.95 , occurs with lattice parameters $(a=b=1, c=0.8, \alpha=\beta=\gamma=\pi / 2)$, where the packing fraction is 0.553 . This tightest unit cell does not give a densest packing: the density increases with the length of the $c$ axis, at the expense of $L / D$, similar to the $\Gamma$ structure.

The $G_{114 R L}^{+}\left(\cosh ^{-1}(2 \sqrt{2})\right)$ weaving, shown in Fig. 10, has tetragonal symmetry $\left(I 4_{1} / a c d\right)$. The structure contains slightly undulating rods, with four distinct axes within a unit cell, described by the vectors $\{u, u,-3 u\},\left\{-u, u, \frac{1}{2}+3 u\right\}$, $\left\{\frac{1}{2}+u, u, \frac{1}{2}+3 u\right\}$ and $\left\{\frac{1}{2}-u, u, 3 u\right\}$. The filaments can be
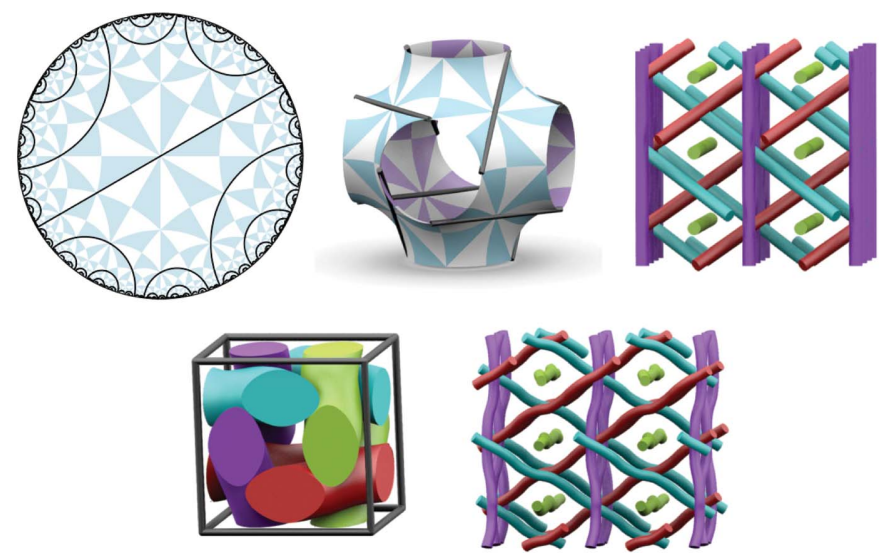

Figure 9

The $P_{114 R L}\left(\cosh ^{-1}(\sqrt{3})\right)$ structure, a tetragonal rod packing composed of straight rods. (Top) The surface structure in $\mathbb{H}^{2}$, on the surface and in $\mathbb{E}^{3}$. (Bottom, left) A unit cell of the ideal embedding of this packing and (right) larger volume of the ideal embedding, drawn with a deflated filament diameter to illustrate the undulating geometry of the rods. 

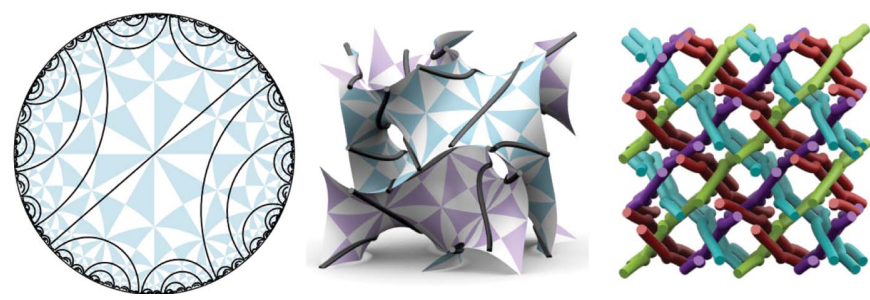

Figure 10

The $G_{114 R L}^{+}\left(\cosh ^{-1}(2 \sqrt{2})\right)$ filamentous array, a tetragonal rod packing composed of slightly undulating filaments. This weaving is shown (left) in $\mathbb{H}^{2}$, (centre) on one unit cell of the $G$ surface and (right) in $\mathbb{E}^{3}$.

straightened to coincide with their axes without changing ambient isotopy, so the $G$ weaving is an equivalent isotope to this new tetragonal rectilinear rod packing. Another distinct class I weaving, formed from the $D_{114 R L}\left(\cosh ^{-1}(3 \sqrt{3})\right)$ tiling, has filaments tracing the same rod trajectories; thus these weavings are equivalent isotopes.

The filaments of the $P_{114 R L}\left(\cosh ^{-1}(5 \sqrt{3})\right)$ weaving can also be rectified to coincide with their axes to give a tetragonal rod packing $(P 4 / n n c)$. The construction of this weaving is shown in Fig. 11. The weaving is composed of slightly undulating filaments, where the four distinct rod positions within a unit cube cell are described by the vectors $\{u, 0,3 u\},\left\{u, \frac{1}{2}, \frac{1}{2}-3 u\right\}$, $\left\{0, u, \frac{1}{2}-3 u\right\}$ and $\left\{\frac{1}{2}, u, 3 u\right\}$.

Similarly, the filaments of the $G_{93 R L}^{+}\left(\cosh ^{-1}(2 \sqrt{2})\right)$ weaving can be rectified to give a rod packing with trigonal symmetry $R \overline{3} c$ (Fig. 12). The three distinct rod positions, described in the $G$ surface cubic unit cell, are given by the vectors $\left\{u, \frac{1}{2}+3 u, \frac{1}{2}+u\right\},\left\{3 u, u, \frac{1}{2}+u\right\}$ and $\left\{u, \frac{1}{2}+u, 3 u\right\}$.

The structure $G_{93 R L}^{-}\left(\cosh ^{-1}(\sqrt{2})\right)$ (Fig. 13) has filament axes forming a rod packing of trigonal symmetry $R \overline{3} c$. The $G_{93 R L}^{-}\left(\cosh ^{-1}(2 \sqrt{2})\right)$ weaving is also an equivalent isotope. The alignment of the rods in a cube cell is described by the vectors $\left\{-u, u, \frac{1}{2}-u\right\},\left\{u, \frac{1}{2}+u, \frac{1}{2}-u\right\}$ and $\left\{\frac{1}{2}-u, u, \frac{1}{2}+u\right\}$ (see Fig. 13). Further, the trigonal rod packing defined by the $G_{93 R L}^{-}\left(\cosh ^{-1}(\sqrt{2})\right)$ weaving has been observed as the arrangement of a self-assembled chemical structure in Carlucci et al. (1999). When viewed along a rod direction, one can see that this weaving is a deformation of the $\Pi^{*}$ cubic rod packing and is thus an isotope of $\Pi^{*}$. Given this, the ideal configuration of the weaving is thus precisely $\Pi^{*}$, for which $L / D=6$ and the packing fraction 0.59 .
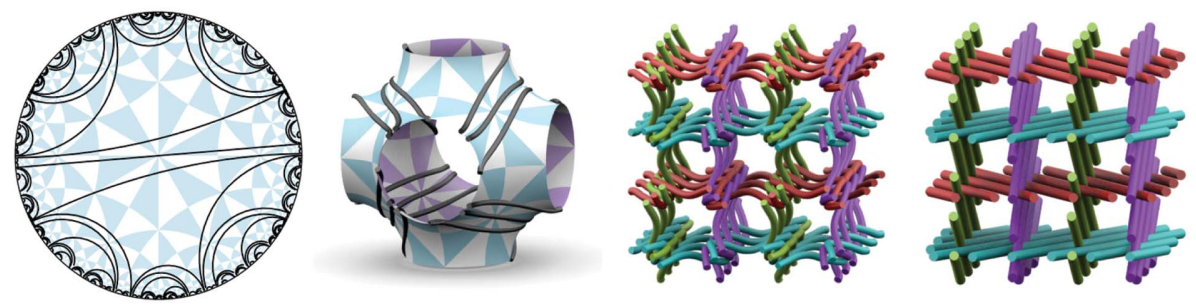

Figure 11

The $P_{114 R L}\left(\cosh ^{-1}(5 \sqrt{3})\right)$ weaving, whose filament axes give a rod packing of tetragonal symmetry $(P 4 / n n c)$. The weaving is shown from left to right as a free tiling of $\mathbb{H}^{2}$, on one unit cell of the $P$ surface, in $\mathbb{E}^{3}$ and where the undulating filaments have been straightened to the associated rod packing.

\section{Class II weavings: intersecting filament axes}

Our enumeration also gives novel class II weavings characterized by intersecting filament axes. Recall that these intersecting filaments form a net whose vertices are the points common to more than one filament. Clearly, ideal embeddings of these weavings necessarily have curvilinear embeddings.

The regular branched-ribbon tiling $P_{118 R L}\left(\cosh ^{-1}(\sqrt{6})\right)$ (Fig. 14) forms a weaving with space group $P 432$ on the $P$ surface. Rectifying the filaments of this weaving along their axes causes the filaments to intersect only when fully straightened. The six distinct filament axes in a cubic unit cell are $\left\{u, \frac{1}{2} \pm u, 0\right\},\left\{0, u, \frac{1}{2} \pm u\right\}$ and $\left\{u, 0, \frac{1}{2} \pm u\right\}$. If the intersection points of the filaments are changed to be vertices, the filaments form a known three-dimensional net, labelled reo according to the three-letter schema developed by O'Keeffe et al. (2008), with 1-transitive vertices and edges. This net is itself a conventional (i.e. not free) tiling of the $P$ (or $D$ ) surfaces, listed as $s q c 877$ in the Epinet database (see http://epinet. anu.edu.au/sqc877).

The $D_{118 R L}\left(\cosh ^{-1}(\sqrt{6})\right)$ weaving has space group $F 4_{1} 32$ on the surface (Fig. 15). The 12 distinct filament axes in a unit cell are $\left\{u, u, \frac{1}{8}\right\},\left\{u, \frac{1}{2}+u, \frac{5}{8}\right\},\left\{u, \frac{1}{8}, u\right\},\left\{u, \frac{5}{8}, \frac{1}{2}+u\right\},\left\{\frac{1}{8}, u, u\right\}$, $\left\{\frac{5}{8}, u, \frac{1}{2}+u\right\},\left\{\frac{7}{8}, u,-u\right\},\left\{\frac{3}{8}, u, \frac{1}{2}-u\right\},\left\{u, \frac{7}{8},-u\right\},\left\{u, \frac{3}{8}, \frac{1}{2}-u\right\}$, $\left\{u,-u, \frac{7}{8}\right\}$ and $\left\{u, \frac{1}{2}-u, \frac{3}{8}\right\}$. Rectification of this weaving results in intersecting rods, giving the crs (cristobalite) net (also known to Epinet as sqc889; see http://epinet.anu.edu.au/ sqc889), which has 1-transitive vertices and edges (O'Keeffe $e t$ al., 2008). The ideal (tight) embedding of this weaving adopts a very low density, with $L / D=145.28$ and a packing fraction of 0.31 .

The $G_{118 R L}^{+}\left(\cosh ^{-1}(\sqrt{6})\right)$ weaving (Fig. 16) is a line pattern on the gyroid with space group $I 4_{1} 32$. The 12 distinct axes of the filaments in a unit cell are $\left\{u, \frac{1}{4}-u, \frac{3}{8}\right\},\left\{u, \frac{1}{4}-u, \frac{7}{8}\right\}$, $\left\{\frac{3}{8}, \frac{1}{4}-u, u\right\}, \quad\left\{\frac{7}{8}, \frac{1}{4}-u, u\right\}, \quad\left\{u, \frac{1}{4}+u, \frac{1}{8}\right\}, \quad\left\{u, \frac{1}{4}+u, \frac{5}{8}\right\}$, $\left\{u, \frac{3}{8}, \frac{1}{4}-u\right\}, \quad\left\{u, \frac{7}{8}, \frac{1}{4}-u\right\}, \quad\left\{\frac{1}{8}, \frac{3}{4}+u, u\right\}, \quad\left\{\frac{5}{8}, \frac{3}{4}+u, u\right\}$, $\left\{u, \frac{1}{8}, \frac{3}{4}+u\right\}$ and $\left\{u, \frac{5}{8}, \frac{3}{4}+u\right\}$. These axes form the chiral nfa net (O'Keeffe et al., 2008), with 1-transitive vertices and 2 -transitive edges. The two distinct edges of the net result from the single asymmetric edge of the weavings because the intersection point of the filaments cuts the asymmetric unit of the filament in half: the two distinct edges of the nfa network together form a continuous straight line. This weaving also has very low density in its ideal conformation, with $L / D=123.84$ and a packing fraction of 0.31 . The difference between the weaving that emerges from the TPMS and the ideal embedding is small.

The $\quad P_{114 R L}\left(\cosh ^{-1}(3 \sqrt{3} / \sqrt{2})\right)$ weaving in Fig. 17 has four distinct filament axes, with vectors $\{u, u,-u\}$, $\left\{u, \frac{1}{2}-u, \frac{1}{2}+u\right\}, \quad\left\{u, u, \frac{1}{2}+u\right\} \quad$ and $\left\{u, \frac{1}{2}-u,-u\right\}$ in a unit cell. It has space group $P 4 / n n c$, and the axes of these filaments form edges of the regular bcu 

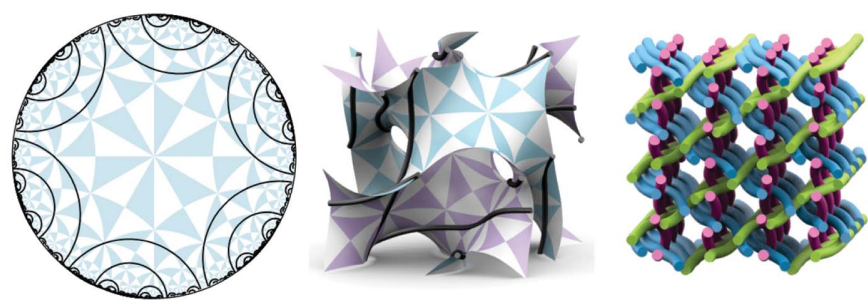

Figure 12

The $G_{93 R L}^{+}\left(\cosh ^{-1}(2 \sqrt{2})\right)$ structure, shown in $\mathbb{H}^{2}$, on the $G$ surface and in $\mathbb{E}^{3}$, is related to a trigonal rod packing.

net (O'Keeffe et al., 2008), known to Epinet as sqc3 (see http:// epinet.anu.edu.au/sqc3).

\section{Class III weavings: tangled examples}

Recall that class III weavings have sufficiently interwoven filaments to impede each other from rectification along their filament axes without changing their entanglement. These examples are therefore 'tangled weavings', since the related rod packing whose (straight) rods lie along filament axes is a distinct isotope. Like class II weavings, ideal embeddings of tangled weavings necessarily contain curvilinear rods.

Fig. 18 shows the $D_{114 R L}\left(\cosh ^{-1}(3 \sqrt{3} / \sqrt{2})\right)$ tiling and resulting weaving. This is a tangled variant of the parallel square rod packing (\#2 rod packing), or in fact any of its equivalent 'canonical' isotopes, which contain all of the parallel and layered invariant rod packings.

The tangled weaving $G_{118 R L}^{+}\left(\cosh ^{-1}(9 / 2)\right)$ is shown in Fig. 19. Rectification of the filaments along their axes results in the
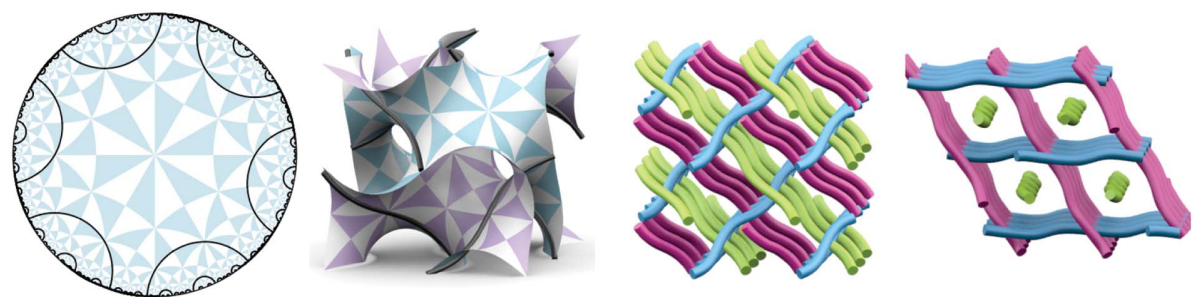

Figure 13

The $G_{93 R L}^{-}\left(\cosh ^{-1}(\sqrt{2})\right)$ structure, shown in $\mathbb{H}^{2}$, on the surface and in $\mathbb{E}^{3}$, is related to a rod packing with trigonal symmetry. The far right image shows the structure when viewed along a rod axis where it can be seen that this is a deformation of the $\Pi^{*}$ rod packing.
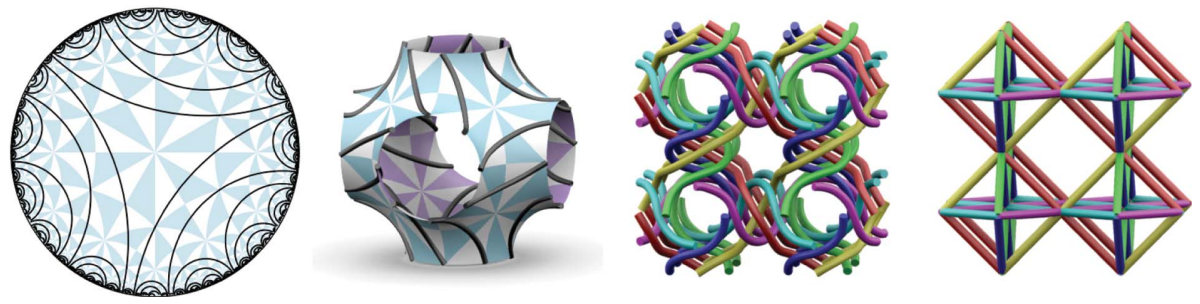

Figure 14

The $P_{118 R L}\left(\cosh ^{-1}(\sqrt{6})\right)$ pattern. (Left to right) The free tiling in $\mathbb{H}^{2}$ and on the $P$ surface, the resulting weaving in $\mathbb{E}^{3}$ and the reo net, whose edges coincide with the filament axes of this weaving.
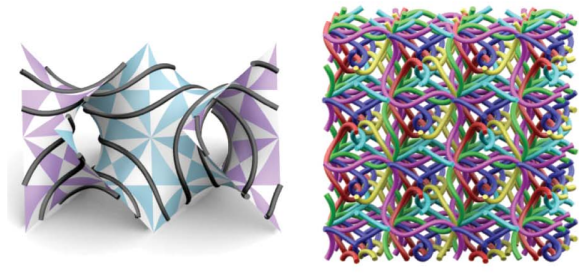

Figure 15

The $D_{118 R L}\left(\cosh ^{-1}(\sqrt{6})\right)$ structure is a weaving with intersecting filament axes. (Top) The free tiling in $\mathbb{H}^{2}$ and on the surface, and the weaving in $\mathbb{E}^{3}$. (Bottom left) The filament axes intersect to form a crs net. (Bottom centre/right) The ideal embedding of this weaving.

$\Gamma$ rod packing with three filaments tracing along each rod. The ideal form of the tangled weaving has $L / D=220.26$ and a packing fraction equal to 0.40 .

The $G_{118 R L}^{-}\left(\cosh ^{-1}(9 / 2)\right)$ weaving, shown in Fig. 20, is a tangled version of the $\Sigma^{+}$rod packing, and hence also the $G_{129 R L}^{+}\left(\cosh ^{-1}(3 / 2)\right)$ weaving ( $c f$. Fig. 6). The ideal conformation of the tangled weaving has $L / D=70.67$ and a packing fraction equal to 0.20 . Its ideal form is the least dense of all weavings constructed so far.

\section{Links: looped filaments}

As noted above, the filament trajectories of the TPMS fibration occasionally form closed loops in $\mathbb{E}^{3}$ rather than infinite lines, resulting in 'links' with an infinite number of components. Many of the examples that emerge from the most symmetric free tilings consist of arrays of disjoint loops, with no entanglement between distinct loops. However, in the case of the tiling $P_{118 R L}\left(\cosh ^{-1}(7 \sqrt{3} / \sqrt{2})\right)$, distinct loops are catenated (Fig. 21). Each loop of the structure catenates 16 of its neighbouring loops, where each loop pair forms a Hopf link (Cromwell, 2004), resulting in a three-dimensional chainmail. Within this structure, sheets of doubly periodic chainmail orient along three orthogonal $\langle 100\rangle$ directions in $\mathbb{E}^{3}$, such that sheets catenate with those of distinct orientations but not with parallel sheets.

The behaviour of this chainmail on tightening using the PB-SONO algorithm is revealing. The link geometry 

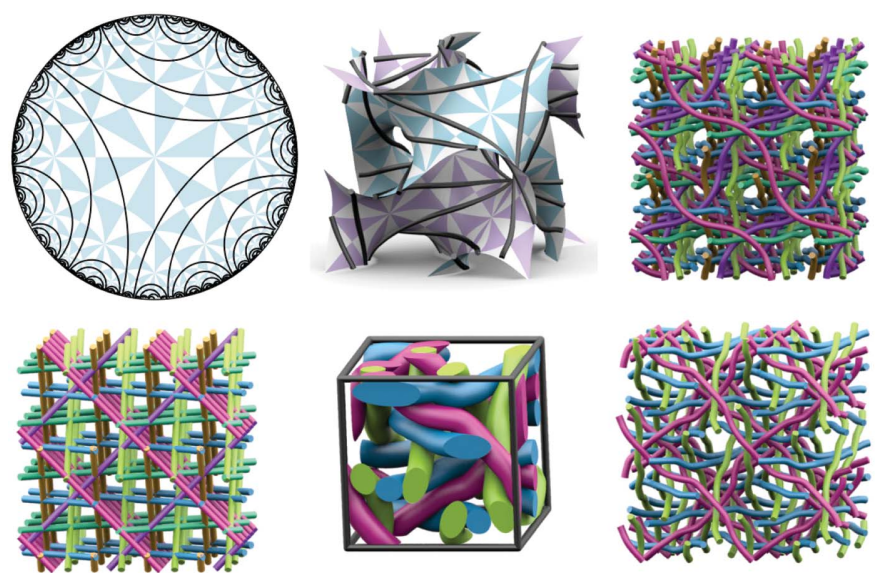

\section{Figure 16}

The weaving $G_{118 R L}^{+}\left(\cosh ^{-1}(\sqrt{6})\right)$. (Top) The regular free tiling in $\mathbb{H}^{2}$ and on one unit cell of the $G$ surface, and the resulting weaving in $\mathbb{E}^{3}$. (Bottom left) The chiral nfa net that results from the intersecting filament axes. (Bottom centre, right) The ideal embedding of the weaving.

inherited from the TPMS tiling has fourfold axes that are lost on tightening. That behaviour is analogous to the ideal embedding of four interwoven helices, where one of the helices straightens along its filament axis and the other three wind around this straight filament, forming a triple helix with a threefold axis. This feature is reminiscent of the ideal configurations of some complex torus knots and links that lose symmetry on tightening (Pieranski, 1998).

\section{Closing remarks}

The wealth of examples derived in this paper, that emerge from the simplest most symmetric line patterns on the simplest TPMS, reveal the efficacy of the construction technique. The process relies on enumeration of free tilings of $\mathbb{H}^{2}$ and we have explored some regular examples in detail. Most of the rod packings described previously from Euclidean crystallographic analysis emerge $a b$ initio in this way. A number of other examples adopt a variety of configurations, leading to our classification of these generic weavings into three possible classes, dependent on their entanglement characteristics. This approach allows us to distinguish between 'tangled' and 'untangled' weavings, by analogy with knots and tangled nets. Most significantly, this enumeration technique allows a natural
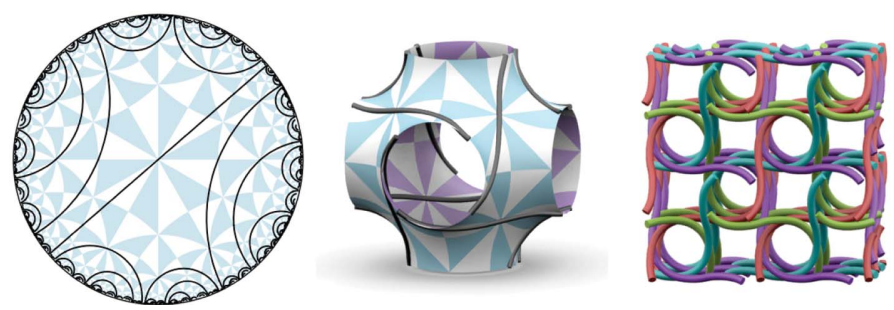

Figure 17

The $P_{114 R L}\left(\cosh ^{-1}(3 \sqrt{3} / \sqrt{2})\right)$ weaving. (Left to right) The free tiling in $\mathbb{H}^{2}$, on one unit cell of the $P$ surface and the resulting weaving in $\mathbb{E}^{3}$. (Right) The bcu net, whose edges coincide with the axes of the filaments in this weaving.
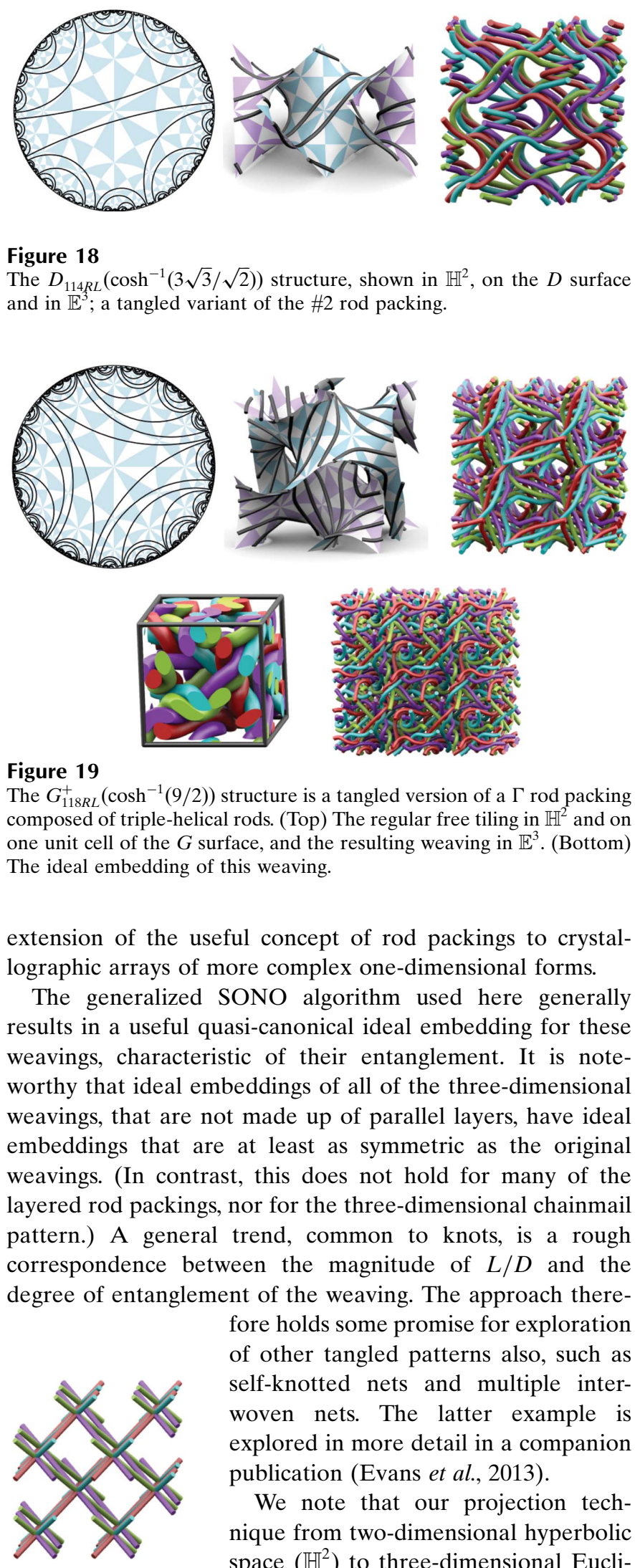

Figure 18

The $D_{114 R L}\left(\cosh ^{-1}(3 \sqrt{3} / \sqrt{2})\right)$ structure, shown in $\mathbb{H}^{2}$, on the $D$ surface and in $\mathbb{E}^{3}$; a tangled variant of the \#2 rod packing.
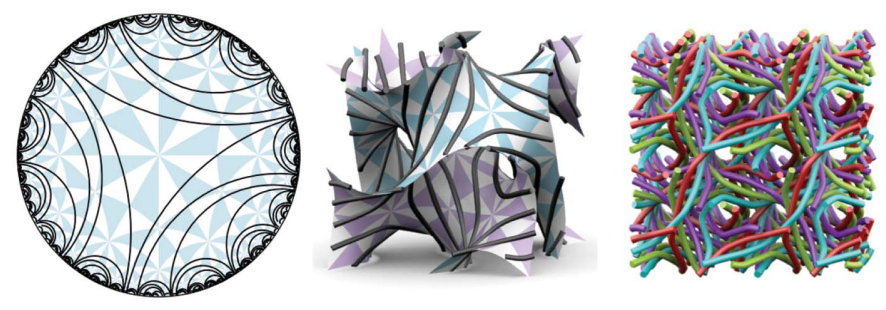

Figure 19

The $G_{118 R L}^{+}\left(\cosh ^{-1}(9 / 2)\right)$ structure is a tangled version of a $\Gamma$ rod packing composed of triple-helical rods. (Top) The regular free tiling in $\mathbb{H}^{2}$ and on one unit cell of the $G$ surface, and the resulting weaving in $\mathbb{E}^{3}$. (Bottom) The ideal embedding of this weaving.

extension of the useful concept of rod packings to crystallographic arrays of more complex one-dimensional forms.

The generalized SONO algorithm used here generally results in a useful quasi-canonical ideal embedding for these weavings, characteristic of their entanglement. It is noteworthy that ideal embeddings of all of the three-dimensional weavings, that are not made up of parallel layers, have ideal embeddings that are at least as symmetric as the original weavings. (In contrast, this does not hold for many of the layered rod packings, nor for the three-dimensional chainmail pattern.) A general trend, common to knots, is a rough correspondence between the magnitude of $L / D$ and the degree of entanglement of the weaving. The approach therefore holds some promise for exploration of other tangled patterns also, such as self-knotted nets and multiple interwoven nets. The latter example is explored in more detail in a companion publication (Evans et al., 2013).

We note that our projection technique from two-dimensional hyperbolic space $\left(\mathbb{H}^{2}\right)$ to three-dimensional Euclidean space $\left(\mathbb{E}^{3}\right)$ occasionally affords a useful embedding for these weavings and little 'annealing' in $\mathbb{E}^{3}$ is required to form the ideal embedding. In other 

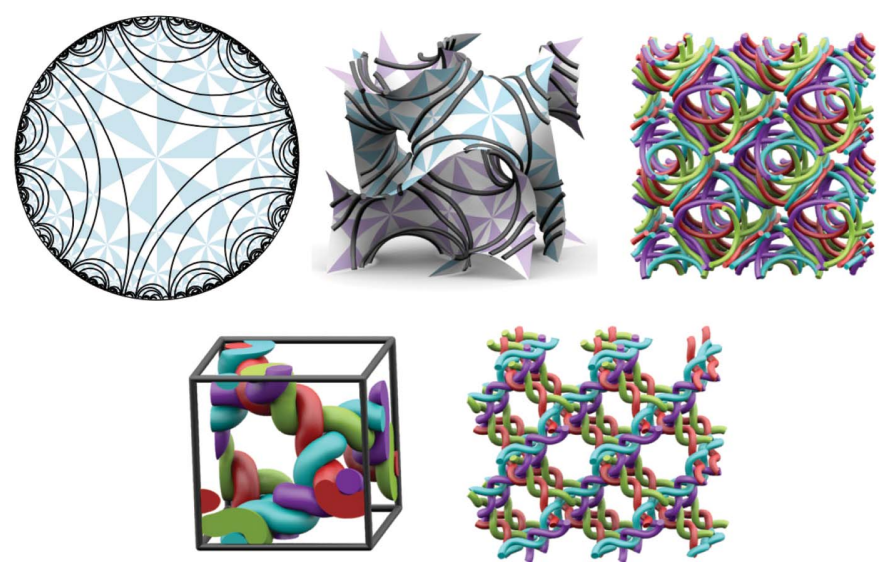

Figure 20

The $G_{118 R L}^{-}\left(\cosh ^{-1}(9 / 2)\right)$ structure is a tangled $\Sigma^{+}$rod packing. (Top) The surface fibration is shown in $\mathbb{H}^{2}$, on one unit cell of the $G$ surface and in $\mathbb{E}^{3}$. (Bottom) The ideal structure.

words, the ideal geometry of the weaving is reminiscent of the TPMS fibration geometry. Certainly, this curvilinear approach is a useful one to generate a variety of weavings, of which rod packings are a subset, since the ideal conformation of some rod packings contains helical filaments rather than straight components.

The paper seeks to enumerate simplest generalized rod packings from the perspective of pure geometry, rather than materials science. However, it is clear that some of the weavings explored here arise in the description of chemical frameworks. In particular, the invariant cubic rod packings are widely identified in covalent inorganic crystal structures and other chemical frameworks (O'Keeffe \& Hyde, 1996). Many of the invariant cubic and non-cubic rod packings are also readily synthesized as metal-organic frameworks, a summary of which may be found in Rosi et al. (2005). Further, the $G_{93 R L}^{-}\left(\cosh ^{-1}(\sqrt{2})\right)$ weaving, shown in Fig. 13, has been observed in the solid state (Carlucci et al., 1999).

These weavings are all constructed as fibrations of surfaces observed regularly as mesoscale cubic membranes. It has been suggested that three-dimensional weavings of structural proteins may form in vivo on the cubic membrane, in much the same way as these weavings emerge as tilings of the TPMS. One such example [the $G_{129 C}^{+}\left(\cosh ^{-1}(3 / 2)\right)$ weaving] was proposed to describe the organization of keratin in the corneocyte of the outer layer of mammalian skin, possibly templated on a G-shaped membrane (Evans \& Hyde, 2011). That weaving has remarkable 'anomalous dilatancy', where straightening of the quasi-helical filaments results in cooperative expansion of the weaving, rather than collapse. This feature is somewhat reminiscent of auxetic (negative Poisson ratio) materials and is characteristic of the (class I) $G_{129 C}^{+}\left(\cosh ^{-1}(3 / 2)\right)$ weaving. It is worth noting that many of the structures from the $G$ surface, and also a few from the $P$ surface, share this property. This construction method then may also be of significance for the design of materials with anomalous expansion characteristics (e.g. negative thermal expansion materials, auxetics). This connection between geometry and topology and mechanical behaviour is curious

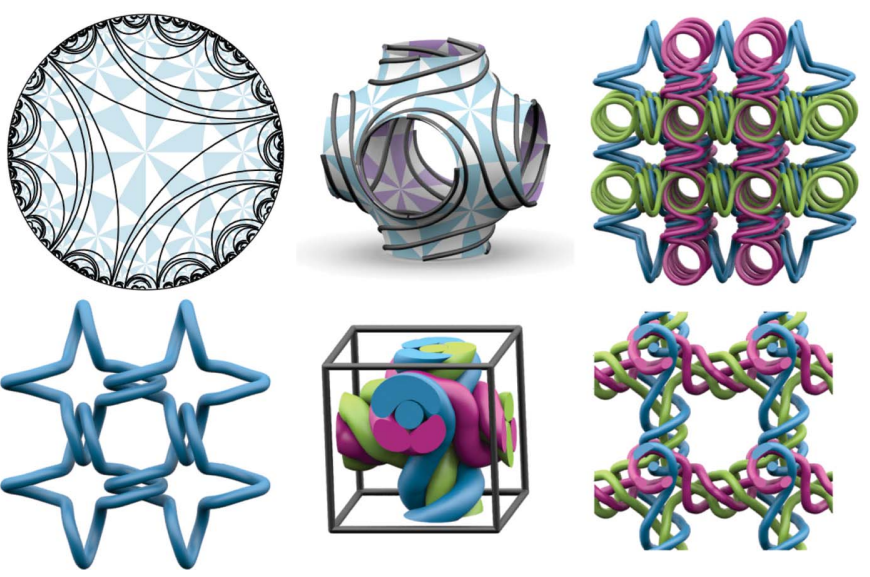

Figure 21

The regular branched-ribbon tiling $P_{118 R L}\left(\cosh ^{-1}(7 \sqrt{3} / \sqrt{2})\right)$ forms complex catenated loops, giving two-dimensional catenated sheets, that catenate orthogonal sheets, giving a three-dimensional chainmail pattern. (Top) The free tiling in $\mathbb{H}^{2}$, on the surface and the resulting chanmail in $\mathbb{E}^{3}$. (Bottom left) Four links within a single layer of the chainmail. (Bottom centre/right) The ideal embedding.

and offers some relevance to the geometric approach introduced here.

\section{APPENDIX $A$ \\ Free tilings of the hyperbolic plane}

The free tilings of $\mathbb{H}^{2}$ with infinite geodesic boundaries are given in Fig. 22. In the companion paper, we detailed free tilings composed of tree-like boundary components, which are the medial axes of the tilings shown in Fig. 22, and vice versa. The edge lengths of each pair of structures differ by virtue of their independent construction; however, we wish to highlight the relation between these structures in the following table.

Geodesic packing
$\star 246_{124 R L}\left(\cosh ^{-1}(\sqrt{3} / \sqrt{2})\right)$
$\star 246_{129 R L}\left(\cosh ^{-1}(3 / 2)\right)$
$\star 246_{118 R L}\left(\cosh ^{-1}(\sqrt{6})\right)$
$\star 246_{118 R L}\left(\cosh ^{-1}(9 / 2)\right)$
$\star 246_{118 R L}\left(\cosh ^{-1}(7 \sqrt{3} / \sqrt{2})\right)$
$\star 246_{118 R L}\left(\cosh ^{-1}(33 / 2)\right)$
$\star 246_{123 R L}\left(\cosh ^{-1}(\sqrt{3} / \sqrt{2})\right)$
$\star 246_{123 R L}\left(\cosh ^{-1}(\sqrt{2})\right)$
$\star 246_{114 R L}\left(\cosh ^{-1}(\sqrt{3})\right)$
$\star 246_{114 R L}\left(\cosh ^{-1}(2 \sqrt{2})\right)$
$\star 246_{114 R L}\left(\cosh ^{-1}(3 \sqrt{3} / \sqrt{2})\right)$
$\star 246_{114 R L}\left(\cosh ^{-1}(3 \sqrt{3})\right)$
$\star 246_{122 R L}\left(\cosh ^{-1}(3 / 2)\right)$
$\star 246_{122 R L}\left(\cosh ^{-1}(5 / 2)\right)$
$\star 246_{93 R L}\left(\cosh ^{-1}(\sqrt{2})\right)$
$\star 246_{93 R L}\left(\cosh ^{-1}(2 \sqrt{2})\right)$

Tree packing (Evans et al., 2013)

$\star 246_{124 R T}\left(\cosh ^{-1}(3)\right)$

$\star 246_{129 R T}\left(\cosh ^{-1}(5)\right)$

$\star 246_{118 R T}\left(\cosh ^{-1}(15)\right)$

$\star 246_{118 R T}\left(\cosh ^{-1}(53)\right)$

$\star 246_{118 R T}\left(\cosh ^{-1}(195)\right.$

$\star 246_{118 R T}\left(\cosh ^{-1}(725)\right)$

$\star 246_{123 R T}\left(\cosh ^{-1}(5)\right)$

$\star 246_{123 R T}\left(\cosh ^{-1}(7)\right)$

$\star 246_{114 R T}\left(\cosh ^{-1}(11)\right)$

$\star 246_{114 R T}\left(\cosh ^{-1}(31)\right)$

$\star 246_{114 R T}\left(\cosh ^{-1}(53)\right)$

$\star 246_{114 R T}\left(\cosh ^{-1}(107)\right)$

$\star 246_{122 R T}\left(\cosh ^{-1}(17)\right)$

$\star 246_{122 R T}\left(\cosh ^{-1}(49)\right)$

$\star 246_{93 R T}\left(\cosh ^{-1}(15)\right)$

$\star 246_{93 R T}\left(\cosh ^{-1}(63)\right)$

\section{APPENDIX $B$ Catalogue of resulting structures}

Tables 1, 2 and 3 show the three-dimensional filament arrays formed from regular branched-ribbon tilings of $\mathbb{H}^{2}$ projected 


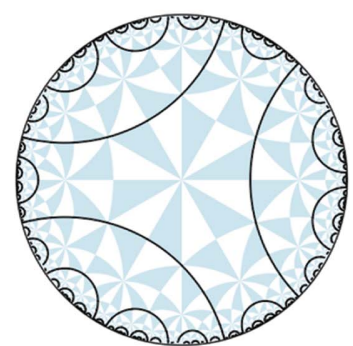

$* 246_{124 R L}\left(\cosh ^{-1}\left(\frac{\sqrt{3}}{\sqrt{2}}\right)\right)$

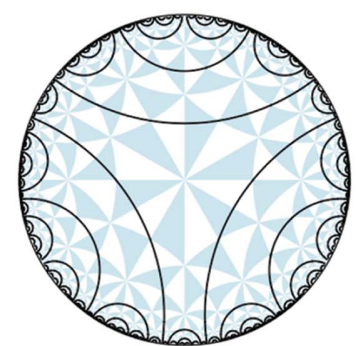

*246 ${ }_{129 R L}\left(\cosh ^{-1}\left(\frac{3}{2}\right)\right)$

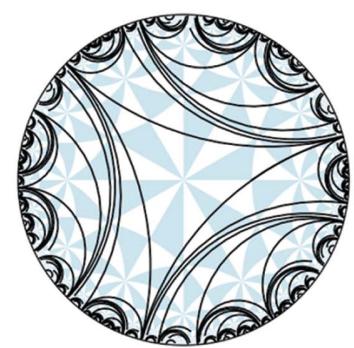

*246 ${ }_{118 R L}\left(\cosh ^{-1}\left(\frac{33}{2}\right)\right)$

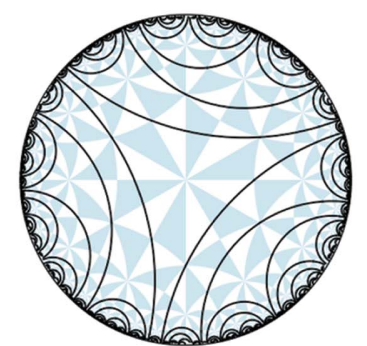

$* 246_{118 R L}\left(\cosh ^{-1}(\sqrt{6})\right)$

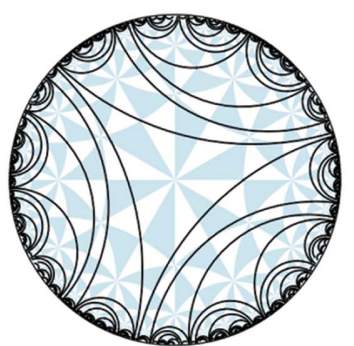

*246 ${ }_{118 R L}\left(\cosh ^{-1}\left(\frac{9}{2}\right)\right)$

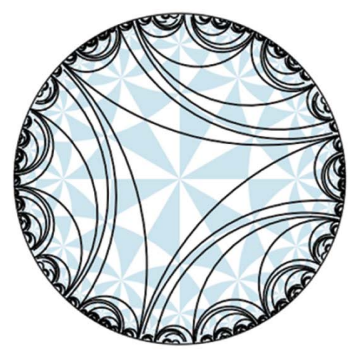

$* 2466_{118 R L}\left(\cosh ^{-1}\left(\frac{7 \sqrt{3}}{\sqrt{2}}\right)\right)$
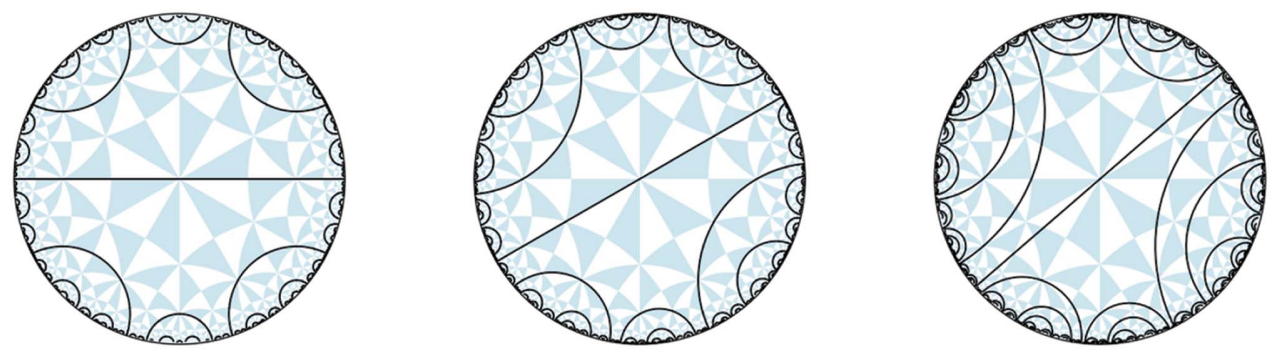

*246 ${ }_{123 R L}\left(\cosh ^{-1}\left(\frac{\sqrt{3}}{\sqrt{2}}\right)\right)$

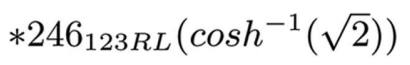

$* 246_{114 R L}\left(\cosh ^{-1}(\sqrt{3})\right)$

*246 ${ }_{114 R L}\left(\cosh ^{-1}(2 \sqrt{2})\right)$
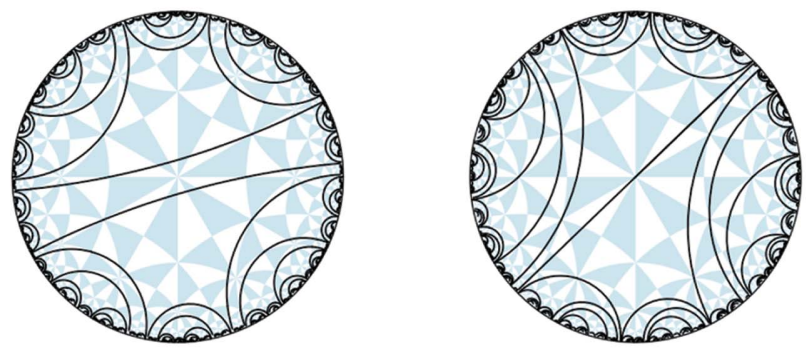

*246 $114 R L\left(\cosh ^{-1}\left(\frac{3 \sqrt{3}}{\sqrt{2}}\right)\right)$

$* 246_{114 R L}\left(\cosh ^{-1}(3 \sqrt{3})\right)$
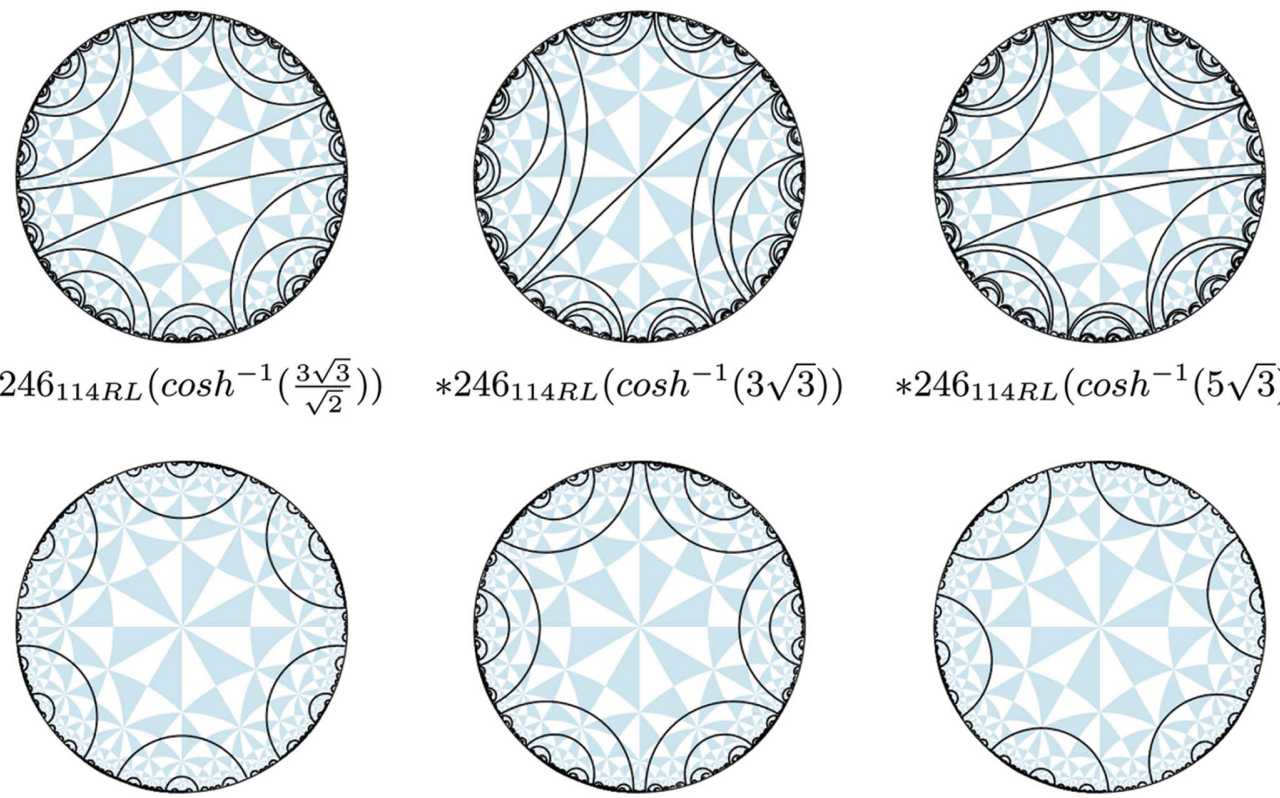

$* 246_{122 R L}\left(\cosh ^{-1}\left(\frac{3}{2}\right)\right)$

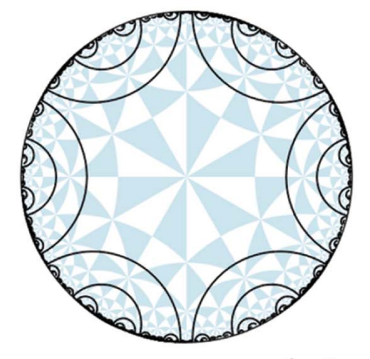

$* 246_{122 R L}\left(\cosh ^{-1}\left(\frac{5}{2}\right)\right)$

$* 246_{114 R L}\left(\cosh ^{-1}(5 \sqrt{3})\right)$

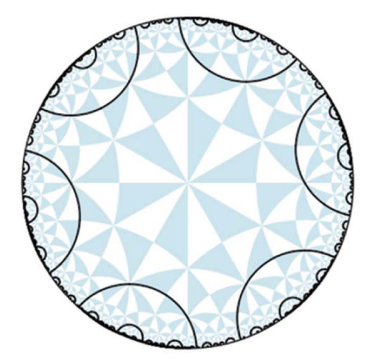

$* 246{ }_{93 R L}\left(\cosh ^{-1}(\sqrt{2})\right)$

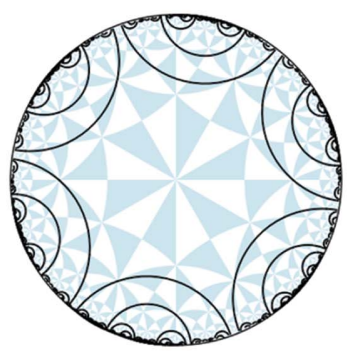

$* 246_{93 R L}\left(\cosh ^{-1}(2 \sqrt{2})\right)$

\section{Figure 22}

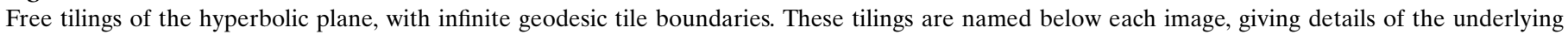
tiling $(\star 246)$, the Delaney-Dress tile topology (see Fig. 1 ), $R L$ to signify regular line packings, and the edge length of one asymmetric unit of the lines. 
Table 1

Weavings from $\star 2223,2 \star 23$ and 2223 hyperbolic symmetry.

\begin{tabular}{|c|c|c|c|c|c|c|}
\hline Structure & Packing & $\begin{array}{l}\text { Weaving } \\
\text { space group }\end{array}$ & $L / D$ & $\begin{array}{l}\text { Packing } \\
\text { fraction }\end{array}$ & Ideal unit cell & Figure \\
\hline$P_{124 R L}\left(\cosh ^{-1}(\sqrt{3} / \sqrt{2})\right)$ & Loops & $\operatorname{Pm} \overline{3} m$ & & & & \\
\hline$P_{129 R L}\left(\cosh ^{-1}(3 / 2)\right)$ & Helical $\Omega^{+}$ & $I 432$ & 24.06 & 0.49 & $(1,1,1, \pi / 2, \pi / 2, \pi / 2)$ & 8 \\
\hline$P_{118 R L}\left(\cosh ^{-1}(9 / 2)\right)$ & Tangled $\Omega^{+}$ & $P 432$ & & & & \\
\hline$P_{118 R L}\left(\cosh ^{-1}(7 \sqrt{3} / \sqrt{2})\right)$ & Catenated loops & $P 432$ & 85.62 & 0.35 & $(1,1,1, \pi / 2, \pi / 2, \pi / 2)$ & 21 \\
\hline$P_{118 R L}\left(\cosh ^{-1}(33 / 2)\right)$ & Tangled $\Omega^{+}$ & P432 & & & & \\
\hline$D_{118 R L}\left(\cosh ^{-1}(\sqrt{6})\right)$ & Intersect & $F 4_{1} 32$ & 145.28 & 0.31 & $(1,1,1, \pi / 2, \pi / 2, \pi / 2)$ & 15 \\
\hline$D_{118 R L}\left(\cosh ^{-1}(9 / 2)\right)$ & Tangled $\Omega^{+}$ & $F 4_{1} 32$ & & & & \\
\hline$D_{118 R L}\left(\cosh ^{-1}(7 \sqrt{3} / \sqrt{2})\right)$ & $\Pi^{*}:$ woven & $F 4_{1} 32$ & & & & \\
\hline$D_{118 R L}\left(\cosh ^{-1}(33 / 2)\right)$ & Tangled $\Omega^{+}$ & $F 4_{1} 32$ & & & & \\
\hline$G_{124 R L}^{+/-}\left(\cosh ^{-1}(\sqrt{3} / \sqrt{2})\right)$ & Helical $\Pi^{+/-}$ & $I 4_{1} 32$ & 17.91 & 0.66 & $(1,1,1, \pi / 2, \pi / 2, \pi / 2)$ & 4 \\
\hline$G_{118 R L}^{-}\left(\cosh ^{-1}(9 / 2)\right)$ & Tangled $\Sigma^{+}$ & $I 4_{1} 32$ & 70.67 & 0.20 & $(1,1,1, \pi / 2, \pi / 2, \pi / 2)$ & 20 \\
\hline$G_{118 R L}^{+}\left(\cosh ^{-1}(7 \sqrt{3} / \sqrt{2})\right)$ & Tangled $\Pi^{+}$ & $I 4_{1} 32$ & & & & \\
\hline$G_{118 R L}^{-}\left(\cosh ^{-1}(7 \sqrt{3} / \sqrt{2})\right)$ & Tangled $\Pi^{+}$ & $I 4_{1} 32$ & & & & \\
\hline$G_{118 R L}^{+}\left(\cosh ^{-1}(33 / 2)\right)$ & Tangled $3 \times \Gamma$ & $I 4_{1} 32$ & & & & \\
\hline$G_{118 R L}^{-}\left(\cosh ^{-1}(33 / 2)\right)$ & Tangled $\Sigma^{+}$ & $I 4_{1} 32$ & & & & \\
\hline
\end{tabular}

Table 2

Weavings from $\star 2224$ and 2224 hyperbolic symmetry.

\begin{tabular}{|c|c|c|c|c|c|c|}
\hline Structure & Packing & $\begin{array}{l}\text { Surface } \\
\text { space group }\end{array}$ & $L / D$ & $\begin{array}{l}\text { Packing } \\
\text { fraction }\end{array}$ & Ideal unit cell & Figures \\
\hline$P_{123 R L}\left(\cosh ^{-1}(\sqrt{3} / \sqrt{2})\right)$ & Loops & $I 4 / \mathrm{mmm}$ & & & & \\
\hline$P_{123 R L}\left(\cosh ^{-1}(\sqrt{2})\right)$ & $\# 2$ & $I 4 / \mathrm{mmm}$ & 1 & 0.90 & $(1,1,1, \pi / 2, \pi / 2, \pi / 3)$ & 2 and 3 \\
\hline$P_{114 R L}\left(\cosh ^{-1}(\sqrt{3})\right)$ & Rod (tetr.) & $P 4 / n n c$ & 15.95 & 0.553 & $(1,1,0.8, \pi / 2, \pi / 2, \pi / 2)$ & 9 \\
\hline$P_{114 R L}\left(\cosh ^{-1}(2 \sqrt{2})\right)$ & Intersect & $P 4 / n n c$ & & & & 17 \\
\hline$P_{114 R L}\left(\cosh ^{-1}(3 \sqrt{3} / \sqrt{2})\right)$ & Helical $4 \times \# 2$ & $P 4 / n n c$ & & & & \\
\hline$P_{114 R L}\left(\cosh ^{-1}(3 \sqrt{3})\right)$ & Tangled (tetr.) & $P 4 / n n c$ & & & & \\
\hline$P_{114 R L}\left(\cosh ^{-1}(5 \sqrt{3})\right)$ & Rod (tetr.) & $P 4 / n n c$ & & & & 11 \\
\hline$D_{123 R L}\left(\cosh ^{-1}(\sqrt{3} / \sqrt{2})\right)$ & $\# 2$ & $\mathrm{P}_{2} / \mathrm{nnm}$ & 1 & 0.90 & $(1,1,1, \pi / 2, \pi / 2, \pi / 3)$ & 2 and 3 \\
\hline$D_{123 R L}\left(\cosh ^{-1}(\sqrt{2})\right)$ & $\# 6$ & $\mathrm{P}_{2} / \mathrm{nnm}$ & 1 & 0.90 & $(1,1,1, \pi / 2, \pi / 2, \pi / 3)$ & \\
\hline$D_{114 R L}\left(\cosh ^{-1}(\sqrt{3})\right)$ & $\Gamma$ & $I 4_{1} /$ acd & 19.27 & 0.71 & $(1,1,1, \pi / 2, \pi / 2, \pi / 2)$ & \\
\hline$D_{114 R L}\left(\cosh ^{-1}(2 \sqrt{2})\right)$ & \#2 & $I 4_{1} /$ acd & 1 & 0.90 & $(1,1,1, \pi / 2, \pi / 2, \pi / 3)$ & \\
\hline$D_{114 R L}\left(\cosh ^{-1}(3 \sqrt{3} / \sqrt{2})\right)$ & Tangled \#2 & $I 4_{1} /$ acd & & & & 18 \\
\hline$D_{114 R L}\left(\cosh ^{-1}(3 \sqrt{3})\right)$ & Rod (tetr.) & $I 4_{1} /$ acd & & & & \\
\hline$D_{114 R L}\left(\cosh ^{-1}(5 \sqrt{3})\right)$ & Tangled $\Gamma$ & $I 4_{1} /$ acd & & & & \\
\hline$G_{123 R L}^{+/-}\left(\cosh ^{-1}(\sqrt{3} / \sqrt{2})\right)$ & $\# 2$ & $I 4_{1} /$ acd & 1 & 0.90 & $(1,1,1, \pi / 2, \pi / 2, \pi / 3)$ & \\
\hline$G_{123 R L}^{+/-}\left(\cosh ^{-1}(\sqrt{2})\right)$ & $\Gamma$ & $I 4_{1} /$ acd & 19.27 & 0.71 & $(1,1,1, \pi / 2, \pi / 2, \pi / 2)$ & 7 \\
\hline$G_{114 R L}^{+}\left(\cosh ^{-1}(\sqrt{3})\right)$ & $\# 2$ & $I 4_{1} /$ acd & 1 & 0.90 & $(1,1,1, \pi / 2, \pi / 2, \pi / 3)$ & \\
\hline$G_{114 R L}^{-}\left(\cosh ^{-1}(\sqrt{3})\right)$ & \#6 & $I 4_{1} /$ acd & 1 & 0.90 & $(1,1,1, \pi / 2, \pi / 2, \pi / 3)$ & \\
\hline$G_{114 R L}^{+}\left(\cosh ^{-1}(2 \sqrt{2})\right)$ & Rod (tetr.) & $I 4_{1} /$ acd & & & & 10 \\
\hline$G_{114 R L}^{-}\left(\cosh ^{-1}(2 \sqrt{2})\right)$ & $\Gamma$ & $I 4_{1} / a c d$ & 19.27 & 0.71 & $(1,1,1, \pi / 2, \pi / 2, \pi / 2)$ & \\
\hline$G_{114 R L}^{+}\left(\cosh ^{-1}(3 \sqrt{3} / \sqrt{2})\right)$ & Helical $3 \times \# 2$ & $I 4_{1} /$ acd & & & & \\
\hline$G_{114 R L}^{-}\left(\cosh ^{-1}(3 \sqrt{3} / \sqrt{2})\right)$ & Tangled \#2 & $I 4_{1} / a c d$ & & & & \\
\hline$G_{114 R L}^{+}\left(\cosh ^{-1}(3 \sqrt{3})\right)$ & Intersect & $I 4_{1} /$ acd & & & & \\
\hline$G_{114 R L}^{-}\left(\cosh ^{-1}(3 \sqrt{3})\right)$ & $\# 2$ & $I 4_{1} /$ acd & 1 & 0.90 & $(1,1,1, \pi / 2, \pi / 2, \pi / 3)$ & \\
\hline$G_{114 R L}^{+}\left(\cosh ^{-1}(5 \sqrt{3})\right)$ & Intersect & $I 4_{1} /$ acd & & & & \\
\hline$G_{114 R L}^{-}\left(\cosh ^{-1}(5 \sqrt{3})\right)$ & Intersect & $I 4_{1} /$ acd & & & & \\
\hline
\end{tabular}

onto the $P, D$ and $G$ (yroid) TPMSs. These weavings are labelled by their parent free tiling ( $c f$. Appendix $A$ ), plus the surface that the tiling decorates. Owing to the pair of embeddings that are possible for chiral patterns on the gyroid
(Robins et al., 2005), $G$ tilings are also labelled with ${ }^{+}$or ${ }^{-}$ superscripts to distinguish these cases. For example, the hyperbolic tiling $* 246_{118 R L}\left(\cosh ^{-1}(\sqrt{6})\right)$ is the embedding of the free tiling with symmetry 2223 (group 118) into the $\star 246$ 
Table 3

Weavings from $2 \star 26$ and 2226 hyperbolic symmetry.

\begin{tabular}{|c|c|c|c|c|c|c|}
\hline Structure & Packing & $\begin{array}{l}\text { Surface } \\
\text { space group }\end{array}$ & $L / D$ & $\begin{array}{l}\text { Packing } \\
\text { fraction }\end{array}$ & Ideal unit cell & Figures \\
\hline$P_{122 R L}\left(\cosh ^{-1}(3 / 2)\right)$ & $\# 1$ & $R \overline{3} m$ & 1 & 0.90 & $(1,1,1, \pi / 2, \pi / 2, \pi / 3)$ & \\
\hline$P_{122 R L}\left(\cosh ^{-1}(5 / 2)\right)$ & Loops & $R \overline{3} m$ & & & & \\
\hline$P_{93 R L}\left(\cosh ^{-1}(\sqrt{2})\right)$ & $\Pi^{*}$ & $R \overline{3} c$ & & & & \\
\hline$P_{93 R L}\left(\cosh ^{-1}(2 \sqrt{2})\right)$ & $\# 1$ & $R \overline{3} c$ & 1 & 0.90 & $(1,1,1, \pi / 2, \pi / 2, \pi / 3)$ & \\
\hline$D_{122 R L}\left(\cosh ^{-1}(3 / 2)\right)$ & Loops & $R \overline{3} m$ & & & & \\
\hline$D_{122 R L}\left(\cosh ^{-1}(5 / 2)\right)$ & $\# 1$ & $R \overline{3} m$ & 1 & 0.90 & $(1,1,1, \pi / 2, \pi / 2, \pi / 3)$ & \\
\hline$D_{93 R L}\left(\cosh ^{-1}(\sqrt{2})\right)$ & Rod (tri.) & $R \overline{3} c$ & & & & \\
\hline$D_{93 R L}\left(\cosh ^{-1}(2 \sqrt{2})\right)$ & Intersect & $R \overline{3} c$ & & & & \\
\hline$G_{122 R L}^{+}\left(\cosh ^{-1}(3 / 2)\right)$ & $\# 1$ & $R \overline{3} c$ & 1 & 0.90 & $(1,1,1, \pi / 2, \pi / 2, \pi / 3)$ & 2 and 3 \\
\hline$G_{122 R L}^{+}\left(\cosh ^{-1}(5 / 2)\right)$ & Helical $2 \times \# 1$ & $R \overline{3} c$ & & & & \\
\hline$G_{93 R L}^{+}\left(\cosh ^{-1}(\sqrt{2})\right)$ & $\# 3$ & $R \overline{3} c$ & 1 & 0.90 & $(1,1,1, \pi / 2, \pi / 2, \pi / 3)$ & 2 and 3 \\
\hline$G_{93 R L}^{-}\left(\cosh ^{-1}(\sqrt{2})\right)$ & Rod (tri.) & $R \overline{3} c$ & 6 & 0.59 & $(1,1,1, \pi / 2, \pi / 2, \pi / 2)$ & 13 \\
\hline$G_{93 R L}^{+}\left(\cosh ^{-1}(2 \sqrt{2})\right)$ & Rod (tri.) & $R \overline{3} c$ & & & & 12 \\
\hline$G_{93 R L}^{-}\left(\cosh ^{-1}(2 \sqrt{2})\right)$ & Rod (tri.) & $R \overline{3} c$ & & & & \\
\hline
\end{tabular}

tiling of $\mathbb{H}^{2}$, embedded with edge length $\cosh ^{-1}(\sqrt{6})$. The structure $G_{118 R L}^{+}\left(\cosh ^{-1}(\sqrt{6})\right)$ is the fibration of the hyperbolic tiling $* 246_{118 R L}\left(\cosh ^{-1}(\sqrt{6})\right)$ over the $G$ surface by one covering map. The space group of the weaving, whose embedding comes from the TPMS, is listed.

We thank Stuart Ramsden for helpful discussions on many aspects of this work. MEE thanks the Humboldt Foundation for generous support.

\section{References}

Carlucci, L., Ciani, G., Macchi, P., Proserpio, D. M. \& Rizzato, S. (1999). Chem. Eur. J. 5, 237-243.

Castle, T., Evans, M. E. \& Hyde, S. T. (2011). Prog. Theor. Phys. Suppl. 191, 235-244.

Castle, T., Evans, M. E., Hyde, S. T., Ramsden, S. J. \& Robins, V. (2012). Interface Focus, 2, 555-566.

Cromwell, P. (2004). Knots and Links. Cambridge University Press.

Evans, M. E. (2011). Three-Dimensional Entanglement: Knots, Knits and Nets. Department of Applied Mathematics, Australian National University.

Evans, M. E. \& Hyde, S. T. (2011). J. R. Soc. Interface, 8, 1274-1280.

Evans, M. E., Robins, V. \& Hyde, S. T. (2013). Acta Cryst. A69, 241261.

Fogden, A. \& Hyde, S. T. (1992). Acta Cryst. A48, 575-591.
Hilbert, D. \& Cohn-Vossen, S. (1952). Geometry and the Imagination. New York: Chelsea Publishing Co.

Hyde, S. T. \& Oguey, C. (2000). Eur. Phys. J. B, 16, 613-630.

O'Keeffe, M. (1992). Acta Cryst. A48, 879-884.

O'Keeffe, M. \& Andersson, S. (1977). Acta Cryst. A33, 914-923.

O'Keeffe, M. \& Hyde, B. G. (1996). Crystal Structures. 1. Patterns and Symmetry. Washington DC: Mineralogical Society of America.

O'Keeffe, M., Peskov, M. A., Ramsden, S. J. \& Yaghi, O. (2008). Acc. Chem. Res. 41, 1782-1789.

O'Keeffe, M., Plévert, J. \& Ogawa, T. (2002). Acta Cryst. A58, 125132.

O'Keeffe, M., Plévert, J., Teshima, Y., Watanabe, Y. \& Ogama, T. (2001). Acta Cryst. A57, 110-111.

Pieranski, P. (1998). Ideal Knots, edited by A. Stasiak, V. Katritch \& L. H. Kauffman, Series on Knots and Everything, Vol. 19, pp. 20-41. World Scientific.

Ramsden, S. J., Robins, V. \& Hyde, S. T. (2009). Acta Cryst. A65, 81108.

Robins, V., Ramsden, S. J. \& Hyde, S. T. (2004). Eur. Phys. J. B, 39, 365-375.

Robins, V., Ramsden, S. J. \& Hyde, S. T. (2005). Eur. Phys. J. B, 48, 107-111.

Rosi, N. L., Kim, J., Eddaoudi, M., Chen, B., O'Keeffe, M. \& Yaghi, O. M. (2005). J. Am. Chem. Soc. 127, 1504-1518.

Stasiak, A., Dubochet, J., Katritch, V. \& Pieranski, P. (1998). Ideal Knots, edited by A. Stasiak, V. Katritch \& L. H. Kauffman, Series on Knots and Everything, Vol. 19, pp. 1-19. World Scientific. 